\title{
Symmetric Phase Only Filtering for Improved DPIV Data Processing
}

\author{
Mark P. Wernet ${ }^{*}$ \\ National Aeronautics and Space Administration \\ Glenn Research Center \\ Cleveland, Ohio, 44135
}

\begin{abstract}
The standard approach in Digital Particle Image Velocimetry (DPIV) data processing is to use Fast Fourier Transforms to obtain the cross-correlation of two single exposure subregions, where the location of the cross-correlation peak is representative of the most probable particle displacement across the subregion. This standard DPIV processing technique is analogous to Matched Spatial Filtering, a technique commonly used in optical correlators to perform the crosscorrelation operation. Phase only filtering is a well known variation of Matched Spatial Filtering, which when used to process DPIV image data yields correlation peaks which are narrower and up to an order of magnitude larger than those obtained using traditional DPIV processing. In addition to possessing desirable correlation plane features, phase only filters also provide superior performance in the presence of DC noise in the correlation subregion. When DPIV image subregions contaminated with surface flare light or high background noise levels are processed using phase only filters, the correlation peak pertaining only to the particle displacement is readily detected above any signal stemming from the DC objects. Tedious image masking or background image subtraction are not required. Both theoretical and experimental analyses of the signal-tonoise ratio performance of the filter functions are presented. In addition, a new Symmetric Phase Only Filtering (SPOF) technique, which is a variation on the traditional phase only filtering technique, is described and demonstrated. The SPOF technique exceeds the performance of the traditionally accepted phase only filtering techniques and is easily implemented in standard DPIV FFT based correlation processing with no significant computational performance penalty. An “Automatic" SPOF algorithm is presented which determines when the SPOF is able to provide better signal to noise results than traditional PIV processing. The SPOF based optical correlation processing approach is presented as a new paradigm for more robust cross-correlation processing of low signal-to-noise ratio DPIV image data.
\end{abstract}

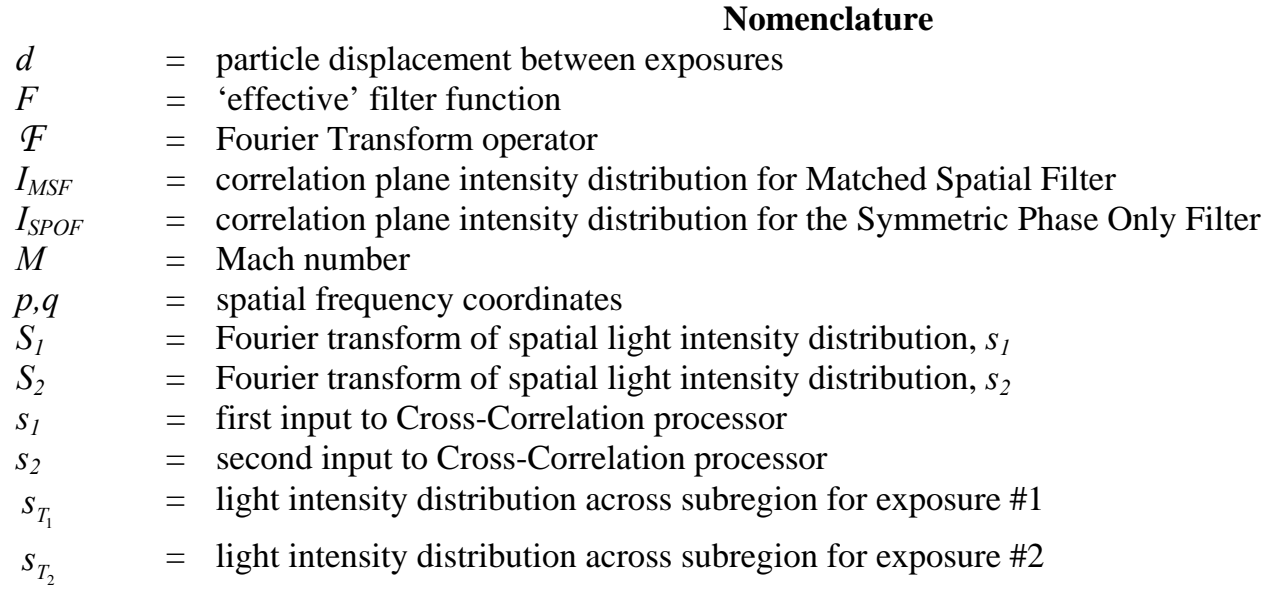

\footnotetext{
* Senior Research Engineer, Optical Instrumentation Technology and NDE Branch, Associate Fellow AIAA
} 


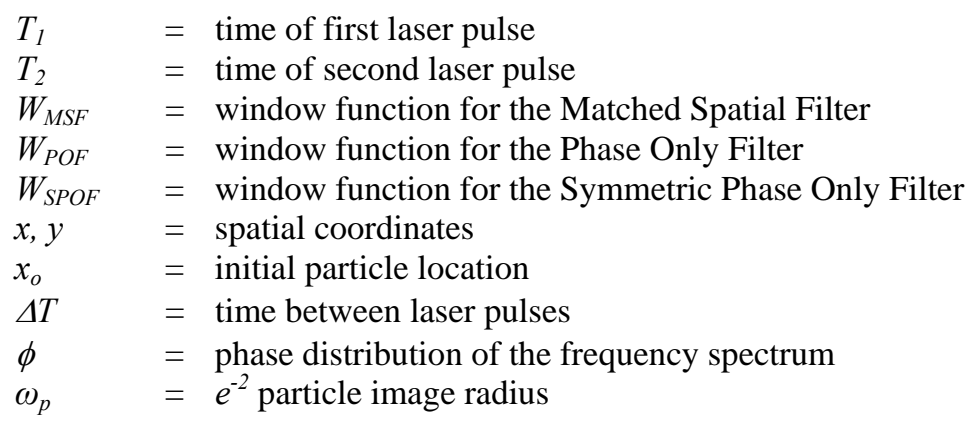

\section{Introduction}

$\mathrm{D}$

PIV has matured into a broadly used and widely accepted planar velocity measurement technique. The use of "frame-straddling" CCD cameras facilitates the use of direct digital processing of the acquired image data. The main components of DPIV image data processing are fairly standardized: FFT based cross-correlation of subregions from the single exposure images yields a correlation peak corresponding to the average displacement of the particles captured at the two instances in time that the laser light sheets are pulsed. Estimating the correlation peak location yields the most probable velocity across the subregion.

In general, DPIV image data obtained from a well seeded flow field are readily processed using traditional DPIV data processing strategies. Such high quality DPIV images are readily obtained in laboratory scale test facilities with tightly controlled environmental conditions. However, as DPIV finds more application in real world, large scale test facilities, image data are frequently acquired that contain non-zero, inhomogeneous background levels, edges or discontinuities in intensity. These discontinuities in recorded intensity can arise from changes in seeding concentration, as is typical in a flame experiment where the seed concentration varies across the flame front; or from a supersonic flow with a shock present; or from a specularly reflecting surface in the field of view of the camera; or even in two-phase flows. Of course, the background noise cannot completely saturate the CCD camera; otherwise no amount of processing will be able to extract the true displacement information. Image segmentation techniques can be used to address some of these problems, but still result in a discontinuity in the processed image at the segmentation boundary, Gui et al. (2003). Background subtraction (provided unseeded flow images have been recorded) can also be used to mitigate any background illumination contaminating the images. However, in some instances the secondary scattered light from the particles in the light sheet illuminates objects or surfaces in the background, making the background subtraction operation less effective. Image processing schemes can also be applied to the raw DPIV image data to improve the correlation result, but such techniques are typically not computationally efficient.

The application of Phase Only Filtering techniques and their benefit to DPIV data processing is discussed in detail by Wernet (2005). In that work, a range of phase only filtering techniques were investigated and it was shown that the Symmetric Phase Only Filter (SPOF) provided the best performance in PIV data processing. In this work we will briefly recap some of the advantages of the SPOF technique and then propose a further refinement in the SPOF processing algorithm, the "Automatic" SPOF.

The Generalized Cross-Correlation (GCC) processor is described, which illustrates the standard DPIV correlation processing steps. Here it is important to realize that the GCC is analogous to Matched Spatial Filtering (MSF). Modification of the matched filter can result in improved correlation plane performance. Conversion of the matched filter into a Phase Only Filter (POF) yields correlation peaks which are significantly sharper and have higher signal to noise ratios than those obtained using traditional DPIV cross-correlation processing techniques. Furthermore, these phase only filters minimize the sensitivity of the DPIV processing technique to flow discontinuities or "edge effects" and more significantly, removes the requirement for background subtraction, even when the image data contain significant non-zero background noise. The phase only filtering techniques do not result in any significant increase in processing time and are easily implemented in existing DPIV processing algorithms.

The superior performance of the phase only filtering techniques will be compared to traditional DPIV processing through a series of test cases. First, FFT processing of a simplified 1-D particle image field is used to obtain the baseline performance of the different filters. Then closed form solutions for the light intensity distribution on the 
correlation plane are computed and compared to the FFT results. The closed form solutions provide insight into why the phase only techniques out perform the matched spatial filtering technique. Next, several DPIV subregion test cases using both synthetically generated and real DPIV image data are used to evaluate the performance of all three filter functions. Finally, the different filtering techniques are used to process real DPIV image data. Through these series of test cases we demonstrate that the SPOF technique is the most robust processing technique for low quality DPIV image data, yielding high accuracy velocity estimates from sharp, high signal to noise ratio correlation peaks. Two different methods for implementing the SPOF algorithm are presented. Symmetric Phase Only Filtering represents a new paradigm, which should be considered for processing all low signal-to-noise ratio DPIV image data, and even high quality DPIV image data.

\section{Generalized Cross-Correlation Description of DPIV Processing}

For clarity, we shall use the concept of a Generalized Cross-Correlation (GCC) processor to illustrate the matched filtering process, as shown in figure 1. There are two inputs to the GCC processor, identified as $s_{I}(x, y)$ and $s_{2}(x, y)$, which are each Fourier transformed. In the lower input path, the complex conjugation operator follows the Fourier transform operator, which is then passed through a windowing operator, $W(p, q)$. The Fourier transformed and windowed inputs are then multiplied together and inverse Fourier transformed. A peak detection operation is performed to determine the correlation peak location.

The GCC processor is analogous to Matched Spatial Filtering, which is commonly used in optical correlators, Vander Lugt (1965). Work in optimizing the performance of optical correlators led to the development of Phase Only Filters and other variants, which form the basis of the filters employed here. In the matched filtering operation, one of the inputs to the GCC is considered the input scene, $s_{I}(x, y)$, and the other is called the 'filter'. When the filter contains an exact replica of the object to be detected in the input scene, it is called a matched filter. As shown in figure 1, the filter is defined here as the product $S_{2}(p, q) W(p, q)$, where $S_{2}(p, q)$ is the Fourier transform of $s_{2}(x, y)$ :

$$
\mathcal{F}\left\{s_{2}(x, y)\right\}=\int_{-\infty}^{\infty} \int_{2} s_{2}(x, y) \cdot e^{-i(p x+q y)} d x d y=S_{2}(p, q)
$$

and $W(p, q)$ is a real valued window function. The spectrum $S_{2}(p, q)$ can also be written as the product of the magnitude and phase information:

$$
S_{2}(p, q)=\left|S_{2}(p, q)\right| \cdot e^{-i \phi_{2}(p, q)}
$$

In the lower input leg of the GCC processor shown in figure 1, the 'effective' filter is then:

$$
F(p, q)=S_{2}(p, q) \cdot W(p, q)
$$

The product of the 'effective' filter function with the Fourier transform of the input scene $S_{l}(p, q)$ yields the filtered spectrum. When the input scene exactly matches the filter function (the matched filter case), then the phase cancels everywhere, yielding a constant, real valued function, which when inverse Fourier transformed, produces a Delta function type response on the output plane.

In the context of the GCC, the filter functions are implemented via the window function $W(p, q)$. In the remainder of the text, the term 'filter' refers to the 'effective' filter combination defined by equation 3 . The GCC processor described and illustrated above is the standard data processing technique used to reduce DPIV image data. In DPIV, the location of a group of particles illuminated by the laser light sheet are recorded on two separate image frames at times $T_{1}$ and $T_{2}=T_{1}+\Delta T$. In the standard DPIV cross-correlation process, we are attempting to determine where the collection of particles recorded at time $T_{1}$ has moved to at time $T_{2}$. The recorded image frames are segmented into small interrogation subregions, where $s_{T_{1}}(x, y)$ is a subregion from image 1 recorded at time $T_{1}$ and $s_{T_{2}}(x, y)$ is a subregion from image 2 recorded at time $T_{2}$. The two interrogation subregions are the inputs to the GCC. In standard DPIV cross-correlation processing (or Matched Filtering Case), the window function $W_{M S F}(p, q)$ is equal to unity, indicating no windowing is performed. The location of the correlation peak on the output plane identifies both the existence of the group of particles recorded at time $T_{1}$ and their location at time $T_{2}$. The position of the correlation peak is then used to determine the average flow velocity across the correlation subregion. Although the 
accepted practice in processing DPIV image data may also include background image subtraction (when these images are available), this processing step is omitted here for simplicity in the subsequent calculations.

In a successful DPIV interrogation region cross-correlation operation, the correlation peak is relatively sharp and has good signal to noise ratio (SNR). Our ability to obtain a velocity estimate depends on detecting the correlation peak above other noise peaks on the correlation plane. Here we will define two types of SNR: $\mathrm{SNR}_{1}$ is the ratio of the correlation peak to the largest amplitude noise peak on the correlation plane, and $\mathrm{SNR}_{2}$ which is the ratio of the energy/unit area in the correlation peak divided by the average energy/unit area on the output plane. Loss of particle pairs from the first to second exposure leads to a decorrelation of the image and a degradation in $\mathrm{SNR}_{1}$

\section{A. Matched Spatial Filter}

The Matched Spatial Filter case exactly replicates traditional DPIV data processing. In the MSF, the window function $W(p, q)$ is unity amplitude and zero phase everywhere:

$$
W_{M S F}(p, q)=1
$$

which when inserted into the GCC, yields the standard DPIV cross-correlation processor. Performance of the $W_{M S F}$ is analogous to results obtained in standard DPIV processing.

\section{B. Phase Only Filters}

Analysis of the Fourier transform of continuous tone pictures has revealed that the phase information is considerably more important than the amplitude information. The amplitude portion of the frequency plane signal contains information more relevant to the size and shape of the object. The phase portion of the frequency plane signal contains the position information of the objects in the input scene and some information regarding their size and shape. POFs have been successfully implemented and demonstrated in optical correlator systems, Horner \& Giannino (1984). In the GCC processor, the POF window function becomes:

$$
W_{P O F}(p, q)=\frac{1}{\left|S_{2}(p, q)\right|}
$$

The 'effective' filter function obtained by substituting equations 2 and 5 into equation 3 yields a purely phase only object, with unity amplitude. The Fourier spectra of finite real valued objects fall off with increasing frequency. The inverse amplitude only window function in equation 5 has a reciprocal response, thereby behaving in a manner similar to a high pass filter. This high pass filtering tends to emphasize edge information, without affecting the phase information. The correlation peaks obtained using POFs in optical correlators are very sharp, since the high spatial frequency information decorrelates very quickly. This high frequency emphasis also makes the POF more sensitive to scale and rotation changes than a conventional MSF. The unity amplitude response of the POF results in broadband noise in the image. This is manifested by an increase in the noise level on the correlation plane. An increase in background noise is acceptable, provided the ratio of the correlation peak to the first highest noise peak remains high.

\section{Symmetric Phase Only Filtering}

All of the traditional matched spatial filtering and phase only filtering techniques were developed for application in optical correlator systems, where the input scene data are acquired optically and processed in real-time. Consequently, the matched filter must be known and prepared a priori in order for the optical correlator to be capable of identifying an object in the input scene. The traditional phase only filtering approach described above is based on modifying the matched filter, and therefore must also be prepared in advance. In the DPIV analogy to matched filtering, we have both the input scene and the filter function recorded and stored before the processing begins. Hence, we have the opportunity to not only modify the properties of the filter function but also the properties of the input scene prior to performing the cross-correlation processing. The Symmetric Phase Only Filtering (SPOF) technique is proposed by the author to provide enhanced processing performance for DPIV image data. In SPOF, the Phase Only Filtering concept of normalizing the filter function by its magnitude is also applied to the input scene. Tests performed by the author have shown that full normalization to unity amplitude (pure phase only objects) of both the input scene and the filter function does not provide optimal results. Instead, the best performance is obtained when the filter and input scene are each normalized by the square root of their respective magnitudes. For the SPOF, the window function becomes: 


$$
W_{S P O F}(p, q)=\frac{1}{\sqrt{\left|S_{1}(p, q)\right| S_{2}(p, q) \mid}}
$$

Hence, by inserting the $W_{S P O F}$ into the GCC processor, we are applying an inverse filter weighting that is balanced according to the signal amplitude content in both $s_{1}(x, y)$ and $s_{2}(x, y)$. The resulting filter is proportional to $|S(p, q)|$ just as in the standard POF case (assuming the filter function and the input scene are identical). In the POF technique, only the filter function $S_{2}(p, q)$ is used to generate the window function $W(p, q)$, which works well if the input scene, $s_{l}(x, y)$ is similar to the filter function. In some cases the input scene and filter function may have different magnitudes and/or content, leading to poor POF performance. Hence, the "balanced" objective of the SPOF approach applies a sufficient amount of normalization to both the input scene spectrum and the filter function spectrum, so that the high frequency content in each is enhanced along with a concomitant reduction in DC content. Implementation of the SPOF is not possible in standard optical correlator systems, but can be easily applied in DPIV cross-correlation processing. We expect the performance of the SPOF to be similar to that of the POF when the input scene is identical to the filter function, however, the advantages of the SPOF technique will be borne out when the content of the input scene and the filter function are different, as found in real DPIV image data.

\section{Automatic Symmetric Phase Only Filter}

As mentioned above, the performance of these various phase only filters has been examined by Wernet, 2005. In this previous study, the SPOF was determined to have the best overall performance when there were stationary objects in the interrogation region or high background noise. However, even though the SPOF yields the best performance in the presence of DC noise in the interrogation region - there are times when the SNR performance of the SPOF is lower than the standard MSF for good quality PIV image data (high number of bright particles on a dark background). The high frequency accentuation feature of the SPOF leads to this decreased SNR performance due to the generation of spurious noise peaks, which can be larger than the normal background noise peaks on the correlation plane from the MSF technique. Rarely, do these spurious noise peaks result in an erroneous velocity estimate in the high image quality regions of the PIV images. In an attempt to obtain an optimal processing scheme, we propose a strategy for determining when the SPOF processing is actually required. Since the MSF yields good performance for interrogation regions containing only particle images (no stationary objects and/or background), and the SPOF yields good SNR correlation peaks in the presence of DC noise - then an optimal processing strategy would be to use the MSF in regions uncorrupted by DC objects and use the SPOF in cases where DC noise is present in the subregion. The only difficulty in implementing this approach is determining when the SPOF is required. SNR criteria are invalid since a high SNR can be computed even from a DC noise dominated subregion (the DC noise peak is the highest peak). The author has determined that the total normalized energy on the correlation plane can be used as a good indicator as to when the SPOF processing would yield a better SNR result than the MSF. The only negative aspect of this approach is the increase in processing time. One additional FFT operation is required to recompute the SPOF, adding a 33\% performance penalty to the overall correlation processing time per subregion where it is required. Obviously this approach is beneficial in cases where the spatial extent of the DC noise is limited in the PIV image data. If the DC noise extends across a large region of the PIV image, then the SPOF technique should just be used everywhere. This automatic SPOF processing approach will be referred to as the ASPOF technique.

In the computation of the correlation plane intensity distribution, the output plane dynamic range is normalized from zero to unity by using the minimum and maximum values on the correlation plane. The total energy on the correlation plane is then computed by summing all of the values across the plane. If the total energy across the correlation plane is higher than a single row of pixels with unity amplitude (equivalent to a normalized energy level equal to the 1-D length of the input correlation subregion size), then the correlation peak and/or the entire correlation plane has a significant amount of energy and is most probably corrupted by DC noise contained in the input correlation subregion. When this energy level threshold is exceeded - the correlation subregion is recomputed using the SPOF algorithm outlined above. Employing this strategy, the optimal processing strategy is applied to the PIV imaged data based on the specific energy content within each processing subregion.

\section{E. Benefits of Phase Only Filters}

The discussion of the POFs above only gives a qualitative description of how they perform better than MSFs. However, this description provides no insight into how the SNR is improved in these phase only techniques. A direct computation of the light intensity distribution on the correlation plane for a simplified DPIV system provides 
additional insight into the performance enhancement offered by the phase only techniques. A 1-D approximation is used here for simplicity, with no loss of generality for the 2-D case. Assume that two Gaussian intensity distributed particle images are recorded on both subregions 1 and 2, which are defined as:

$$
s_{1}(x)=e^{\frac{-2\left(x \pm x_{0}\right)^{2}}{\omega_{p}^{2}}} \text { and } s_{2}(x)=e^{\frac{-2\left(x \pm x_{0}+d\right)^{2}}{\omega_{p}^{2}}}
$$

where $\omega_{p}$ is equal to $e^{-2}$ particle image radius. The particles are initially located at $\pm x_{0}$ at the first laser pulse and then move to a location $\pm x_{0}+d$ at the time of the second laser pulse.

The expected correlation plane result is obtained in two ways: by direct computation using FFTs and by an exact analytic solution. The results for the MSF and POF are shown in figure 2, where all of the correlation peaks have been normalized to 1 . The SPOF case is omitted since the result is the same as the POF for this ideal case where the input scene and the filter function are identical. The $\mathrm{SNR}_{1}$ values for the two filter functions are: $\mathrm{MSF}=4.0, \mathrm{POF}=$ 9.0. Both results have sidelobes located at $\pm 2 x_{0}$. The sidelobes originate from the partial correlation of the two particle images recorded on each single exposure image used in this example (particle\#1 in exposure \#1 crosscorrelating with particle\#2 in exposure \#2 and vice versa). The correlation peak and sidelobe widths are narrower in the POF case.

The closed form solution for the light intensity distribution for the MSF and POF cases are computed and additional details can be found in Wernet (2005). All of the steps defined by the GCC processor were performed on the input subregions including the $W_{M S F}$ filter function. The intensity distribution on the output plane for the Matched Spatial Filter case is:

$$
I_{M S F}(x)=\frac{\omega_{p}^{2} \pi}{4}\left[4 e^{\left(\frac{-2(x+d)^{2}}{\omega_{p}^{2}}\right)}+e^{\left(\frac{-2\left(x+d+2 x_{0}\right)^{2}}{\omega_{p}^{2}}\right)}+e^{\left(\frac{-2\left(x+d-2 x_{0}\right)^{2}}{\omega_{p}^{2}}\right)}\right]
$$

The closed form solution for the MSF is plotted in figure 3 along with the result obtained from the FFT computation. The correlation peak is located at $x=d$ and the sidelobes are located at $x=d \pm 2 x_{0}$. The ratio of the correlation peak to the first noise peak (or sidelobe) for the MSF is 4, which agrees with the result obtained using the FFT to compute the MSF SNR value.

For the Phase Only Filter case, we insert $W_{P O F}$ into the GCC processor and obtain the following intensity distribution:

$$
I_{P O F}(x)=\frac{1}{4} \sum_{n=-\infty}^{\infty} e^{\frac{-4\left(x-d-\frac{n}{2 x_{0}}\right)^{2}}{\omega_{p}^{2}}}\left[\operatorname{Sinc}\left(\frac{x-d}{2 x_{0}}+\frac{1}{2}\right)+\operatorname{Sin}\left(\frac{x-d}{2 x_{0}}-\frac{1}{2}\right)\right]^{2}
$$

where $\operatorname{Sinc}(x)=\sin (\pi x) / \pi x$. The POF correlation plane intensity distribution is described by an infinite sum of Gaussians, located at integer multiples of the spacing between the particle images, modulated by the sum of two Sinc functions. Both the FFT estimate of the POF correlation plane intensity distribution and the POF closed form solution are plotted in figure 4, and match identically. For reference, the sum of the two Sinc functions envelope is also shown in the figure. Three main observations can be made, based on the closed form solutions, regarding the POF result compared to the MSF result. The first is that the POF correlation peak amplitude is lower in magnitude and is independent of the input particle size. This is the feature that makes the POF insensitive to DC objects in the input subregion. In the Phase Only Filtering correlation process, all objects receive the same weighting. The DC information has only a single component (it occurs only once in the subregion). The particle displacement correlation peak is computed from the sum of the contributions from the alignment of all of the particle images across the subregion. Hence, the particle displacement peak can now exceed the amplitude of the DC noise peak (provided there is more than one particle pair across the subregion). The second observation is that the Gaussian peaks in the POF correlation function are narrower than those in the MSF result. The correlation peak widths are at least $\sqrt{2}$ smaller than the MSF result and never wider than the sum of the two Sinc functions. The third observation is that the POF correlation plane result (correlation peak and infinite train of sidelobes) is modulated by the sum of two Sinc functions, which is the main reason for the significantly higher $\mathrm{SNR}_{1}$. The width of the Sinc function 
scales directly with the particle displacement and inversely with the particle separation in the subregion. The Sinc function significantly attenuates the sidelobes on the correlation plane. The infinite sum in equation 9 does lead to more sidelobes on the correlation plane; however, their amplitudes are severely attenuated. This infinite sum is the source of the increased broadband noise anticipated from accentuating the high frequency content in the image. When the spacing between particles on the subregion is large (low seeding concentration) then the Sinc function will be broadened, yielding less attenuation of the sidelobes and possibly a lower $\mathrm{SNR}_{1}$. Note that the POF result exactly predicts the SPOF performance for the idealized input used here.

One final comment is required regarding the applicability of phase only filtering to cross-correlation processing. The phase only techniques demonstrated above result in a large correlation peak with significantly attenuated sidelobes. The phase only filtering techniques offer no performance benefit in auto-correlation processing of multiple exposure DPIV image data. In auto-correlation processing, it is the sidelobes that contain the particle displacement information. Hence, the use of phase only filtering techniques would yield auto-correlations with poor qualities for estimating the particle displacement correlation peak location.

\section{Application of Phase Only Filtering Techniques to DPIV}

In a normal Matched Spatial Filtering operation (standard DPIV cross-correlation processing) the correlation peak is a maximum when the particle images line up between the two interrogation regions. The recorded particle images nominally have a Gaussian distributed intensity distribution. In standard cross-correlation processing, the width of the correlation peak is determined by the spatial extent of the objects being correlated (particle images). Large particle images yield large diameter correlation peaks. The nominal correlation peak diameter is approximately $\sqrt{2} d_{p}$, where $d_{p}$ is the nominal particle image diameter across the subregion, Wernet and Pline, (1993). The correlation peak width is also affected by velocity gradients across the correlation subregion, being elongated perpendicular to the direction of the gradient.

In some instances, there are DC objects in the field of view of the DPIV system that cannot be removed or eliminated. These DC objects can be surfaces or test objects, surface flare light, ambient background light, or background light at the laser frequency. Image contamination by ambient light is most prevalent in the second exposure when using frame-straddling cameras, which is typically 2 orders of magnitude longer than the first image exposure period. If the DC noise saturates the camera and completely encompasses the correlation subregion, then no information can be extracted. Here we are considering cases where the DC noise is below the level of the particle images or, if surface flare light, it does not encompass the entire correlation subregion. When there are DC objects in the correlation subregion, then the resulting DPIV correlation plane result takes on a shape that is representative of the convolution of the DC object with the particle images, for example, a triangle function for a hard edge. Stationary objects in both the first and second DPIV image exposures have only magnitude information, with zero phase shift, which yields a large amplitude, broad DC peak at the origin of the correlation plane. The large, broad DC peak at the origin often dominates the correlation plane, completely masking the particle displacement peak, resulting in an invalid measurement.

The performance of the phase only filters is determined mostly from the object position information, and to a lesser extent on the shape of the objects. In the cross-correlation operation, as soon as the 'center' positions of the particles no longer overlap, the correlation peak goes to zero. Hence the phase only filter based correlation peaks are very sharp. The phase only filters are insensitive to the large amplitude contributions from edges or static objects in the correlation subregion. Factors that contribute to broadening the MSF based correlation should also affect the phase only filters. For example, the existence of a velocity gradient across a correlation subregion should yield a slightly broadened correlation peak when using a phase only filter, which was verified by Wernet, 2005.

While phase only filters cannot perform as well as MSFs under all conditions, when the amplitude information dominates the frequency space complex conjugate product, the phase only filter performance is superior to that of the MSF. As in the example above, if there is an edge in the DPIV image and that edge spans a subregion, then the resulting correlation plane will contain the correlation peak corresponding to the net shift of the particle images from exposure \#1 to exposure \#2, in addition to the Fourier transform of the edge, centered at the origin on the correlation plane (no net displacement between exposures 1 and 2). In most instances, the ringing from the edge dominates the output plane with a large amplitude broad peak centered at the origin. However, in the phase only filter case, the 
DC peak is severely attenuated, enabling the particle displacement peak to be detected above the residual noise, hence yielding a valid measurement where it was previously not possible.

\section{Evaluation of Filter Functions for DPIV}

The performance of these filters on DPIV image data were evaluated here using three test cases:

Case 1) Computer generated DPIV image data;

Case 2) Experimental DPIV data from a centrifugal compressor with surface flare light

Case 3) Experimental DPIV image data from a flame front region, showing two distinct particle seed densities across the flame front

The author recognizes the limited usefulness of computer generated particle image data. Accurate evaluation of any processing technique involving optically recorded data requires inclusion of the Poisson noise and other image recording noise sources in the computer generated particle image subregions. Case 1 was used to ensure that the filter functions were returning the true particle displacements and that no biases were being introduced by using the phase only based techniques. Cases 2 and 3 use experimental data with all of the noise sources inherent in the DPIV technique: Poisson noise, camera read noise, variation in particle size, non-zero background level.

The simulated DPIV image data were generated with a Gaussian intensity distribution, yielding 3 pixel wide particle images in the subregion. The Case 2 DPIV data was obtained from a centrifugal compressor experiment, where surface flare light from a diffuser vane is contaminating the subregion. The nominal particle size was $0.7 \mu \mathrm{m}$ and a $1 \mathrm{kx} 1 \mathrm{k}$ pixel, 8-bit CCD sensor was used to record the images. The experimental image data used in Case 3 was obtained from a low turbulence flame experiment. The seed particles are $0.5 \mu \mathrm{m}$ diameter alumina particles and the images were recorded using a $2 \mathrm{kx} 2 \mathrm{k}$ pixel, 8 bit cross-correlation camera. The data used in Case 3 is from the flame front region, where the upper left portion of the subregion is inside the flame zone and the lower right portion of the image is in the cooler, denser ambient air. The concentration of seed particles changes dramatically across the flame front due to both consumption of seed particles and the significant decrease in density between the combusting and non-combusting regions. The results from all of the test cases are summarized in Table I. The correlation peak $\mathrm{e}^{-2}$ widths were estimated by fitting the correlation peak intensity distribution to a Gaussian function, which were then used to compute the $\mathrm{SNR}_{2}$ values in the table.

The results from Case 1, computer generated image data, are shown in figure 5 for all three filter types: 5a) MSF; 5b) POF; and 5c) SPOF. The first exposure for the simulated input data subregion is shown at the top left of the figure. Both a pixilated inverse gray scale map and a 3-D perspective plot of the output correlation plane and are shown for each filter type. The actual correlation plane origin is located at coordinate $(16,16)$ in all of these plots. The correlation peak is observed to become narrower and the signal to noise ratio higher when phase only filtering is applied, as seen in figures 5b-5c. The noise peaks for the MSF in 5a are small compared to the correlation peak with a $\mathrm{SNR}_{1}$ of 30.7. For the POF case, the noise peaks are barely discernable and the $\mathrm{SNR}_{1}$ value is 181 . The SPOF result is identical to the POF case at an $\mathrm{SNR}_{1}$ of 181. All three filter functions returned the actual particle displacement. Refer to Table I for the narrower correlation peak widths for the phase only filtering cases compared to the MSF result.

The Case 2 results for the centrifugal compressor data with surface flare light from a diffuser vane surface are shown in figure 6. The diffuser vane surface is delineated by the bright line running nearly horizontally at the top of the subregion in the top left of figure 6 . The results for the three filter functions are very different. For the MSF filter, the output correlation plane is dominated by the DC energy contained in the surface flare light, which is manifested as 3 horizontal ridges. There is marginal evidence of a correlation peak in the MSF result. The true correlation peak has an $\mathrm{SNR}_{1}$ value of 0.98 , meaning it is not the highest peak on the correlation plane and may not be detected. The energy from the surface flare light masks the true correlation peak location. This result is exemplary to that obtained using traditional DPIV processing techniques. The POF result also contains three horizontal ridges, but of much lower amplitude. The correlation peak is clearly observed above the surface flare light energy in the central ridge, having an $\mathrm{SNR}_{1}$ value of 3.87. The correlation peak displacement measured using the POF for this case is (-8.7, 1.7), and appears essentially free of any bias from the filtering or the DC contamination in the subregion. The SPOF result is shown in figure 6c. The SPOF correlation plane has much lower energy ridges than the POF case in figure 6b. The SNR 1 value for the SPOF case is 4.44 , again better than the POF case. Here we see the true value of the phase only filters since they are able to suppress the DC content in the interrogation subregion. 
The Case 3 results for the experimental DPIV data in the flame front region are shown in figure 7, where two distinct fluid densities (and seeding densities) are observed across the subregion. The results obtained here are similar to the Case 2 results. In figure 7a, the MSF output is dominated by the 'edge' effect caused by the discontinuity in particle seeding across the subregion. The output correlation plane contains slight evidence of a correlation peak; however, the peak is buried in the side of the large DC peak at the origin of the correlation plane. The POF result in figure $7 \mathrm{~b}$ shows a clear correlation peak with an $\mathrm{SNR}_{1}$ of 1.43 . The SPOF case is slightly better having the highest $\mathrm{SNR}_{1}$ value of 1.90. The correlation displacement peak is essentially the same for both of the phase only filtering cases.

Based on the 3 test cases above, all of the phase only filtering techniques appear to offer better performance (higher $\mathrm{SNR}_{1}$ values and narrower correlation peaks) than the MSF. Of the Phase Only Filtering techniques, the SPOF technique had the highest $\mathrm{SNR}_{1}$ most of the time and appears to provide the greatest noise suppression when the DC content in the subregion is high. Although indicative of their relative performance, the $\mathrm{SNR}_{1}$ values do not conclusively determine the optimum filtering approach for actual DPIV image data, since a spurious noise peak can result in a lower $\mathrm{SNR}_{1}$ value. The $\mathrm{SNR}_{2}$ values give a better perspective on the overall performance, since it is the ratio of the energy density in the correlation peak to the energy density in the remainder of the correlation plane. A high value of $\mathrm{SNR}_{2}$ indicates a processing approach that yields low noise across the entire subregion, not just relative to the second largest peak. Comparing the $\mathrm{SNR}_{2}$ values, we see that the POF and SPOF techniques have the highest values. More tests are required in order to determine the best performing technique. In the next section we will apply the different filtering techniques to actual DPIV image data to determine the best performing approach.

\begin{tabular}{|c|c|c|c|c|c|c|}
\hline & & $\mathrm{SNR}_{1}$ & $\mathrm{SNR}_{2}$ & $\begin{array}{l}\text { Peak } \\
\text { X-width }\end{array}$ & $\begin{array}{l}\text { Peak } \\
\text { Y-width }\end{array}$ & Peak Location \\
\hline \multirow{3}{*}{$\begin{array}{l}\text { Case 1: } \\
\text { Simulated Data }\end{array}$} & MSF & 30.7 & 146.6 & 2.96 & 2.92 & $4.0,2.0$ \\
\hline & POF & 181 & 2311. & 2.12 & 2.12 & $4.0,2.0$ \\
\hline & SPOF & 181 & 2293. & 2.12 & 2.12 & $4.0,2.0$ \\
\hline \multirow{3}{*}{$\begin{array}{l}\text { Case 2: } \quad \text { Exper. } \\
\text { Data Surface Flare }\end{array}$} & MSF & 0.98 & - & - & - & - \\
\hline & POF & 3.87 & 322. & 2.16 & 2.16 & $-8.788,1.781$ \\
\hline & SPOF & 4.44 & 290. & 2.28 & 1.96 & $-8.802,1.776$ \\
\hline \multirow{3}{*}{$\begin{array}{l}\text { Case 3: Seeding } \\
\text { Interface }\end{array}$} & MSF & - & - & - & - & - \\
\hline & POF & 1.43 & 12.5 & 2.84 & 2.20 & $5.338,0.359$ \\
\hline & SPOF & 1.90 & 12.9 & 2.96 & 2.32 & $5.297,0.328$ \\
\hline
\end{tabular}

Table I. Performance summary of the different filter functions for the 3 test cases.

\section{Application to Real DPIV Image Data}

A true test of the phase only filtering techniques can only be conclusively determined by testing them on real DPIV image data. Hundreds of different DPIV images were used to validate the performance of the phase only filtering techniques. In all cases where flare light was present, the phase only filters performed better than the MSF. However, in standard high signal to noise DPIV image data the performance of the different phase only techniques was not always better than the MSF, although the correct displacements were always found. Three test cases will be discussed here: The first comparison is based on the DPIVChallenge 2001 vortex image A001 supplied by Kähler, as described in Stanislas, et al. (2003), and the second and third test case images are from stereo DPIV experiments involving high speed (M 0.9), heated axi-symmetric and chevron nozzle flows. The vortex image was selected since it is representative of a classic DPIV image, is publicly available and has a background noise level that steadily increases towards the bottom of the image. The 3-D DPIV nozzle flows were selected because the light sheet was oriented in the cross-stream direction, yielding images containing significant surface flare light contamination from the model. The two image test cases were processed using the MSF, POF and SPOF. Of the phase only filtering techniques, the best performing technique was the SPOF. The ratio of the SPOF map of SNR 1 to the MSF map of $\mathrm{SNR}_{1}$ is shown in figure 8a. The pixilated gray scale maps of the ratio of $\mathrm{SNR}_{1}$ values are only plotted from the range of 0.98 to 2, to best delineate the regions where the $\mathrm{SNR}_{1}$ of the SPOF technique exceeds that of the MSF processing technique. Values below 1 are set to pure white. In addition to the $\mathrm{SNR}_{1}$ ratios, the ratio of the $\mathrm{SNR} 2$ 
values for the SPOF to those of the MSF are plotted in figure 8b. Again, any white regions are areas where the MSF processing yielded a higher $\mathrm{SNR}_{1}$ or $\mathrm{SNR}_{2}$ value than the corresponding symmetric phase only filtering technique. The 0.98 cutoff is used to account for round off errors in the computations of the ratios. The SPOF technique yields higher $\mathrm{SNR}_{1}$ values than the MSF technique $60 \%$ of the time with an average ratio of 1.31 . The SPOF technique yields a lower $\mathrm{SNR}_{1}$ value than the MSF technique $40 \%$ of the time where the average ratio is 0.78 . Although, the SPOF $\mathrm{SNR}_{1}$ values are lower in some regions, the correct correlation peak is still readily identified. The lower $\mathrm{SNR}_{1}$ values are caused by spurious secondary noise peaks, which happen to be larger than the secondary noise peaks in the MSF cases. For the ratio of the $\mathrm{SNR}_{2}$ values, the SPOF nearly always outperforms the MSF. An interesting feature in figure $8 \mathrm{~b}$ is the effect of the increasing background noise in the bottom $20 \%$ of the image. The ratio of $\mathrm{SNR}_{2}$ values in this region are consistently higher, again confirming the superior performance of the SPOF technique compared to the MSF in low signal to noise ratio image data.

The second DPIV image test case is shown in figure 9. The central portion of a sample image from a stereo DPIV experiment is shown in figure 9a. The flow field is from a $50 \mathrm{~mm}$ diameter heated nozzle flow at $\mathrm{M}$ 0.9. The light sheet is oriented perpendicular to the nozzle flow and is located 0.1 nozzle diameters downstream from the nozzle exit. Secondary scattered light from particles in the plane of the light sheet illuminates the nozzle in the recorded DPIV image data. The nozzle has been painted black to reduce the amount of scattered light. Background image subtraction is not possible in this case since it is predominately the secondary scattered light from the flow seeding that illuminates the nozzle. Standard DPIV cross-correlation processing (MSF case) of the nozzle flow is shown in figure 9b, where the flare light from the nozzle lip in the bottom and top of the nozzle core flow results in spurious velocity vectors. Figure 9c shows the computed velocity field using the SPOF technique, where the effects of the nozzle flare light have been eliminated.

Standard DPIV cross-correlation processing (MSF case) of a sequence of 200 image frame pairs from both the left and right camera views from the stereo PIV setup were used to compute the ensemble averaged, 3-component velocity field, of which only the w-component is shown in figure 10a. The contour plot of the w-component of velocity reveals the resulting surface flare contamination from the left and right camera views in the final 3component velocity field. Figure 10b shows the same ensemble averaged velocity field using the SPOF processing technique. No evidence of the surface flare light is observed in the final 3-component velocity field. Hence, the SPOF is the best technique for processing DPIV image data that have poor signal to noise or surface flare light contamination.

The third example case is shown in figure 11. The stereo DPIV data are from a $50 \mathrm{~mm}$ diameter, heated chevron nozzle flow at M 0.9. The light sheet is again oriented in the cross-stream direction and located 0.1 nozzle diameters downstream from the nozzle exit. Although the model is painted black, the secondary scattered light from particles in the light sheet illuminates the model, clearly delineating the chevrons. The left and right camera views are shown along with the images warped back to the fluid space. The warped, overlapping image pair illustrates the level of model flare in the raw PIV images. Figure 12 shows the resulting ensemble averaged velocity field for this data set. A sequence of 200 velocity vector maps were ensemble averaged to compute the 3-component velocity field. Only the w-component of velocity is shown here. The plot on the left was obtained using standard PIV processing techniques (MSF), where the flare light off of the nozzle chevrons has corrupted the lower region of the computed nozzle flow field. The plot on the right was generated from PIV data processed using the SPOF technique. No evidence of the nozzle flare light remains in the processed velocity map.

The results of these tests confirm that the SPOF technique is the preferred data processing approach for DPIV image data. The SPOF yields high SNR correlation peaks $100 \%$ of the time when the correlation subregions are contaminated by DC noise. For high quality PIV image data (no DC noise contamination) the SPOF yields higher $\mathrm{SNR}_{1}$ values $60 \%$ of the time and correlation peaks with an $\mathrm{SNR}_{1}$ value an average of $78 \%$ of the MSF correlation peak $\mathrm{SNR}_{1}$ the remaining $40 \%$ of the time. Implementing the SPOF technique in the standard correlation based PIV processing algorithm results in a 5\% increase in the processing time for a given PIV image. For DPIV image data with background noise contamination, the SPOF technique will yield the highest SNR correlation peaks. Therefore, the author presents the SPOF technique as the DPIV processing technique of choice when marginal quality DPIV data are to be processed. If the DPIV image data are of high quality, then standard MSF (cross-correlation) processing or SPOF can be used. The SPOF technique generally produces sharper, higher $\mathrm{SNR}_{1}$ correlation peaks than the standard DPIV processing techniques. The SPOF technique should also be considered if the particle images are large or streaked - since the correlation peaks will be independent of the particle image sizes and shapes. 


\section{Automatic SPOF}

From the tests on the DPIVChallenge image set in the previous section we learned that the SPOF can generally outperform the standard MSF technique. However, the SPOF technique is not necessarily always required - since some of the time, in high quality particle image cases, the MSF outperforms the SPOF. Rarely does the SPOF generate an erroneous vector where the MSF produces a correct vector. However, a more optimal strategy would employ the SPOF technique only when the background flare light or DC noise in the correlation subregion was large enough to significantly impact the MSF correlation result. In Section II D., we described a strategy for implementing an "Automatic" SPOF technique. The technique is based upon the total energy on the correlation plane, where the correlation result is normalized to range from zero to unity using the correlation minimum and maximum values. A good quality DPIV correlation results in a large, narrow correlation peak on the correlation plane. Hence, the total energy level in this case is relatively low. However, if the correlation is dominated by DC noise, then the correlation plane will contain a large, broad correlation peak, or peaks. These large correlation peaks will contain significantly more energy than the narrow correlation peaks from a high quality correlation. A map of the correlation plane total energy for the Stereo PIV data from the axi-symmetric nozzle is shown in figure 13a. In this figure we see that the total energy is nearly always below 45 . Only in the regions where there is flare light scattered off of the nozzle does the energy level exceed 45 . Figure $13 \mathrm{~b}$ shows the same total energy plot with values below 64 blanked out. Here we clearly see where the SPOF is actually needed to improve the SNR in the correlation result.

Using a threshold level of 64, the Automatic SPOF technique was used to process the axi-symmetric nozzle data. The ratio of the ASPOF to the MSF $\mathrm{SNR}_{1}$ and $\mathrm{SNR}_{2}$ values are shown in figure 14 . The MSF is used primarily throughout the image, yielding the large uniform gray regions (i.e., ratio=1.0) in the plot. In the regions near the nozzle exit, the SPOF technique has been applied to increase the $\mathrm{SNR}_{2}$ values. The few times that the SPOF must be invoked - and the correlation result recomputed - do not significantly add to the overall processing time. The velocity vector map obtained using the Automatic SPOF is essentially the same as that obtained using the SPOF. Fewer spurious vectors are detected in the periphery of the image, since the MSF is used in these regions.

\section{Peak Locking Test}

The SPOF yields sharper correlation peaks with higher SNR $_{1}$ than the traditional MSFs, or standard DPIV processing. The higher $\mathrm{SNR}_{1}$ values obtained using phase only filters improves the robustness of the particle displacement estimate. The accuracy of the DPIV measurement is related to the correlation peak width. If the peak is too narrow - then a "peak-locking" effect may exist, Westerweel (1997). A simple comparison test was performed on the DPIVChallenge 2001 vortex image A001. The image was processed using the MSF, and SPOF based correlation operations employing multi-pass, integer subregion-offset processing. The first pass processing was $64 \times 64$ pixels and the second pass was 32x32 pixels, all at 50\% overlap. Histograms of the u-component of velocity are shown in figure 15 . The MSF result is in figure 15a and shows evidence of peak locking. The SPOF result is shown in figure 15b and has only a slight amount of peak locking. From these results, it appears that the phase only filtering techniques provide sharper correlation peaks, but do not appear to increase the peak locking effect, and may possibly reduce the peak locking effect. The reason for the lower level of "peak locking" in the SPOF result is probably due to the fact that the SPOF technique effectively removes any background in the image. Background image subtraction in standard PIV processing can reduce the amount of "peak locking" in a given PIV image, Westerweel (2000). Lack of a suitable background image for the A001 image data prevented background subtraction and may be a factor in the higher "peak locking” effect observed in the MSF case.

\section{Conclusion}

Cross-correlation based DPIV image processing is analogous to the matched spatial filtering technique used in optical correlator systems. Phase only filtering is a variation of the matched spatial filtering concept, which offers superior performance by generating sharper, higher SNR correlation peaks. Application of the phase only filtering technique to DPIV image data was shown to provide similar improvements in the DPIV correlation peak properties. Sharper correlation peaks are obtained without introducing "peak locking”. Correlation peaks spanning 1-2 pixels yield the highest accuracy DPIV velocity estimates. Hence, the narrow correlation peaks obtained using the phase only filtering techniques results in increased accuracy velocity estimates. 
Closed form solutions for the MSF and phase only filtering techniques illustrated the factors contributing to the decreased sidelobe energy on the correlation plane when using the phase only techniques. In addition to the decreased sidelobe energy, the phase only filtering techniques were shown to be insensitive to the amplitude information of the objects in the interrogation subregion. Since all objects in the interrogation region have equal weighting, the amplitude of the correlation peak or noise peaks is determined by their relative frequency of occurrence in the subregion. A noise object (surface flare light) exists only once in the subregions. However, particle images occur many times and the cumulative energy obtained by summing over all of the matched particle image pairs across the first and second exposure subregions yields a large correlation peak relative to the noise peaks. It is this insensitivity to input signal amplitude that enables the phase only filtering techniques to produce readily identifiable correlation peaks in low quality PIV image data cases previously not possible using standard DPIV data processing techniques. Image masking and background subtraction are not required in order to obtain valid velocity measurements in contaminated image data, thereby simplifying and expediting the data processing operation. The "effective" background removal obtained using the phase only filtering techniques also results in a reduced level of peak locking and therefore more accurate velocity estimates. Image data obtained with large particle images can also be accurately processed using the phase only filtering techniques resulting in sharper correlation peaks and hence, high accuracy velocity estimates.

In this work, the performance of the Symmetric Phase Only Filtering (SPOF) technique was investigated and compared to the traditional cross-correlation DPIV processing technique. The new SPOF technique, described herein, was shown to have the better performance on actual DPIV image data than the MSF technique. The SPOF technique supersedes the capabilities of the existing phase only filtering techniques by operating on both the filter function and the input scene, for more "balanced" filtering. The SPOF technique only marginally outperforms the MSF technique for high quality image data. However, when the DPIV image data are contaminated with background noise, the SPOF technique significantly outperforms the MSF technique, producing high quality correlation peaks where the MSF technique actually fails. Implementing the full SPOF technique in conventional cross-correlation based PIV processing algorithms results in a 5\% increase in the processing time. An "Automatic" SPOF algorithm was also presented where the MSF is used to process the image and the energy content on the output correlation plane is monitored to determine the extent of the background noise in the correlation subregion. When the detected background noise level exceeds a preset level, then the SPOF is used to reprocess the correlation subregion in order to produce a high quality correlation peak. The SPOF technique represents a new paradigm which should be considered for all DPIV image data processing.

\section{References}

Gui, L., Wereley, S. T., Kim, Y. H., 2003, “Advances and Applications of the Digital Mask Technique in Particle Image Velocimetry Experiments”, Meas. Sci. Technol. 14, pp. 1820-1828.

Horner, J. L. and Giannino, P. D., 1984, "Phase only Matched Filtering”, Appl. Opt., 23, No. 6, pp. 812-816.

Stanislas, M., Okamoto, K., Kähler, C., 2003, “Main Results of the First International DPIV Challenge”, Meas. Sci. Technol., 14 pp. R63-R89.

Vander Lugt, A., 1964, "Signal Detection by Complex Spatial Filtering” IEEE Trans. Inform. Theory, Vol. IT-10, pp. 139-145.

Wernet, M. P. and Edwards, R. V., 1988, “Application of Optical Correlation Techniques to Particle Image Velocimetry”, NASA TM 101306.

Wernet, M. P. and Pline, A., 1993, "Particle Displacement Tracking Technique and Cramer-Rao Lower Bound Error in Centroid Estimates From CCD Imagery”, Exper. in Fluids 15, pp. 295-307.

Wernet, M. P., 2005, "Symmetric Phase Only Filtering: a New Paradigm for DPIV Data Processing”, Meas. Sci. Technology, 16, pp. 601-618.

Westerweel, J., 1997, “Fundamentals of Digital Particle Image Velocimetry”, Meas. Sci. Technol., 8, pp.1379-1392.

Westerweel, J., 2000, “Theoretical Analysis of the Measurement Precision in Particle Image Velocimetry”, Exp. Fluids, 29, pp. S3-S12. 


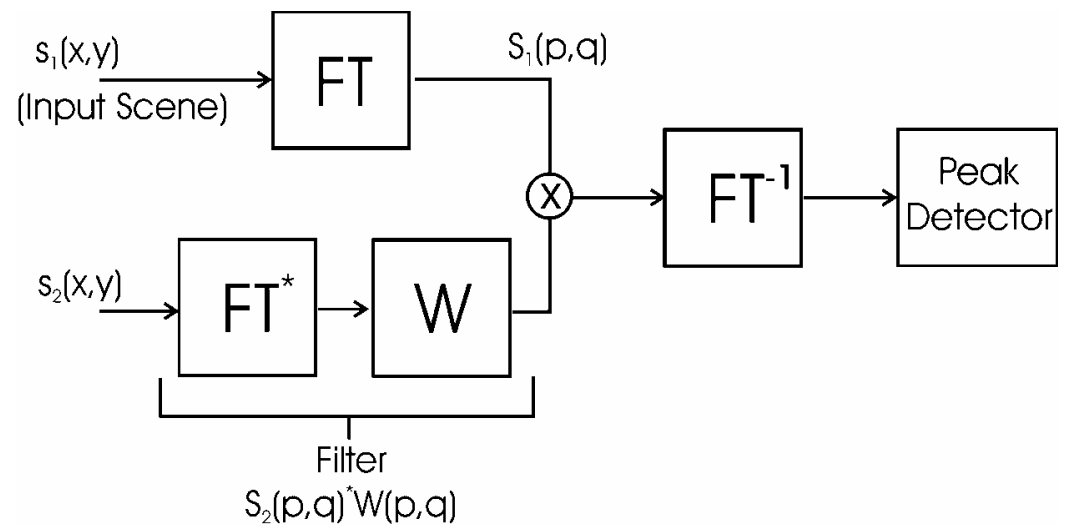

Figure 1: Generalized Cross-Correlation processor where the filter function is generated by the product of the $S_{2}(p, q)$ and $W(p, q)$. The filter is used to detect the presence of $s_{2}(x, y)$ in the input scene, $s_{1}(x, y)$. Changing the properties of the window $W$ can improve the performance of the filter.

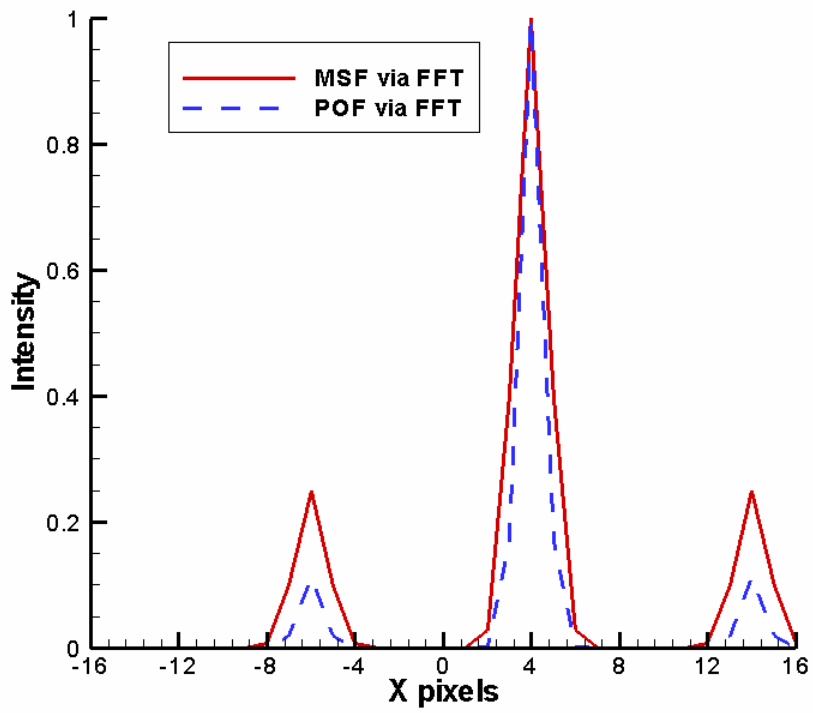

Figure 2: Output correlation plane computed using FFT for the MSF and POF filters for a simulated 1-D two particle input field.

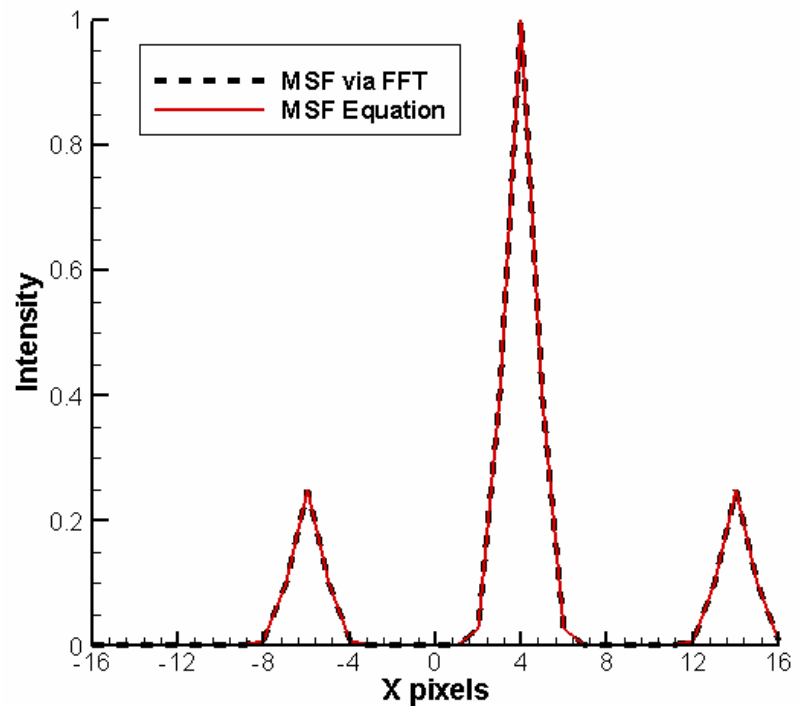

Figure 3: Comparison of the FFT computed output and the closed form solution for the MSF case.

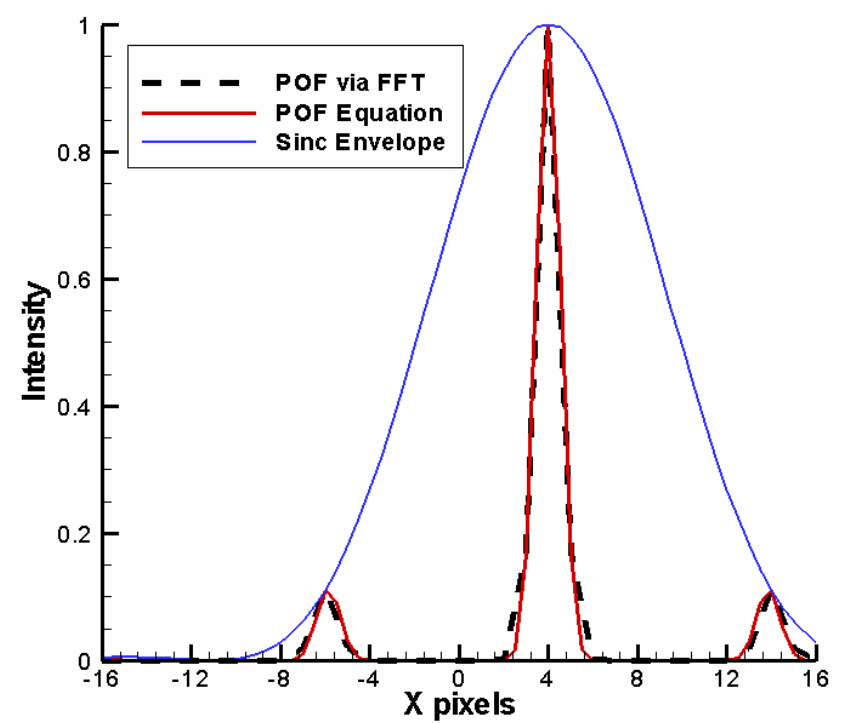

Figure 4: Comparison of the FFT computed output and the closed form solution for the POF case. The Sinc ${ }^{2}$ envelope is included for reference. 

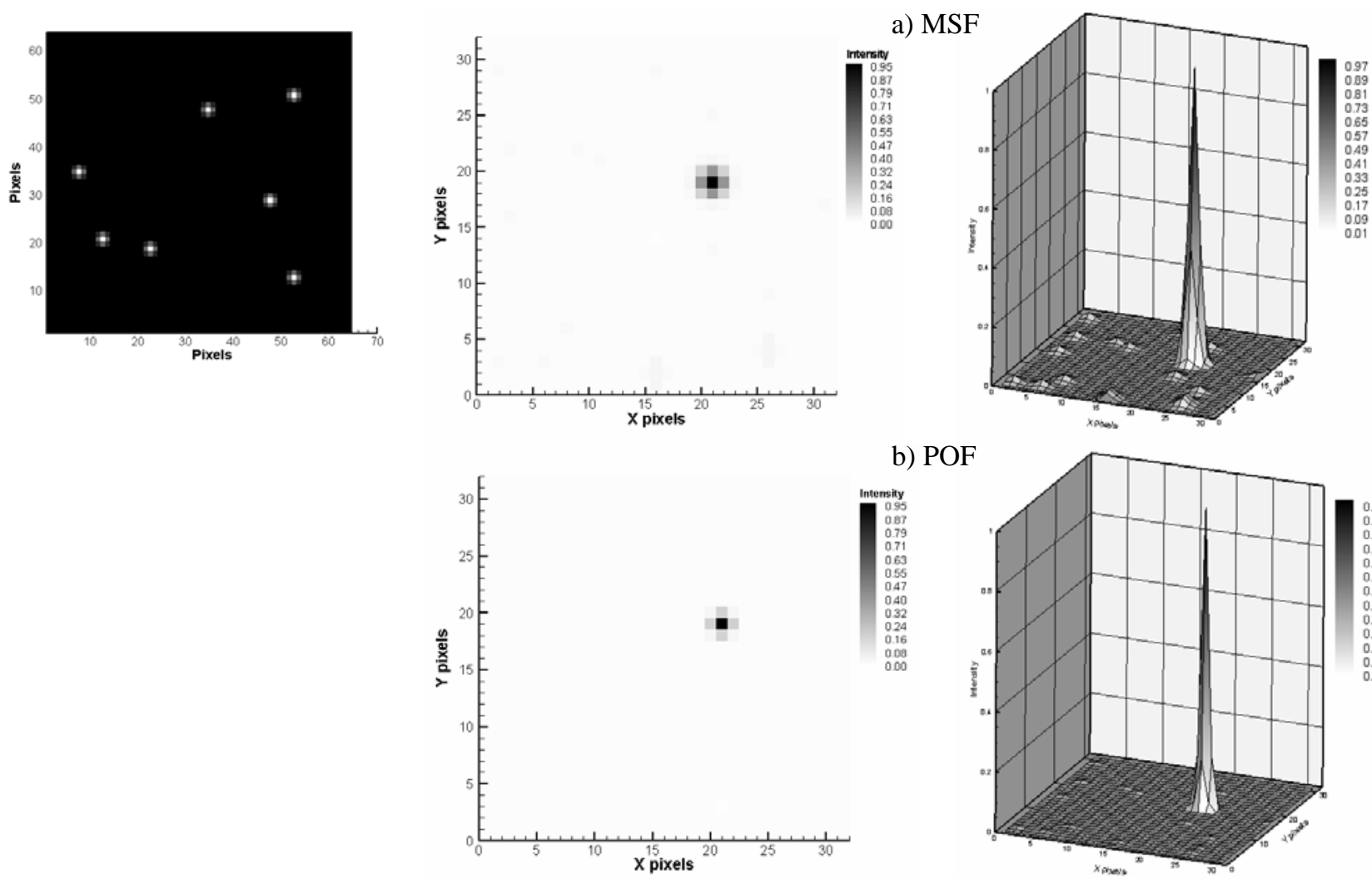

b) POF
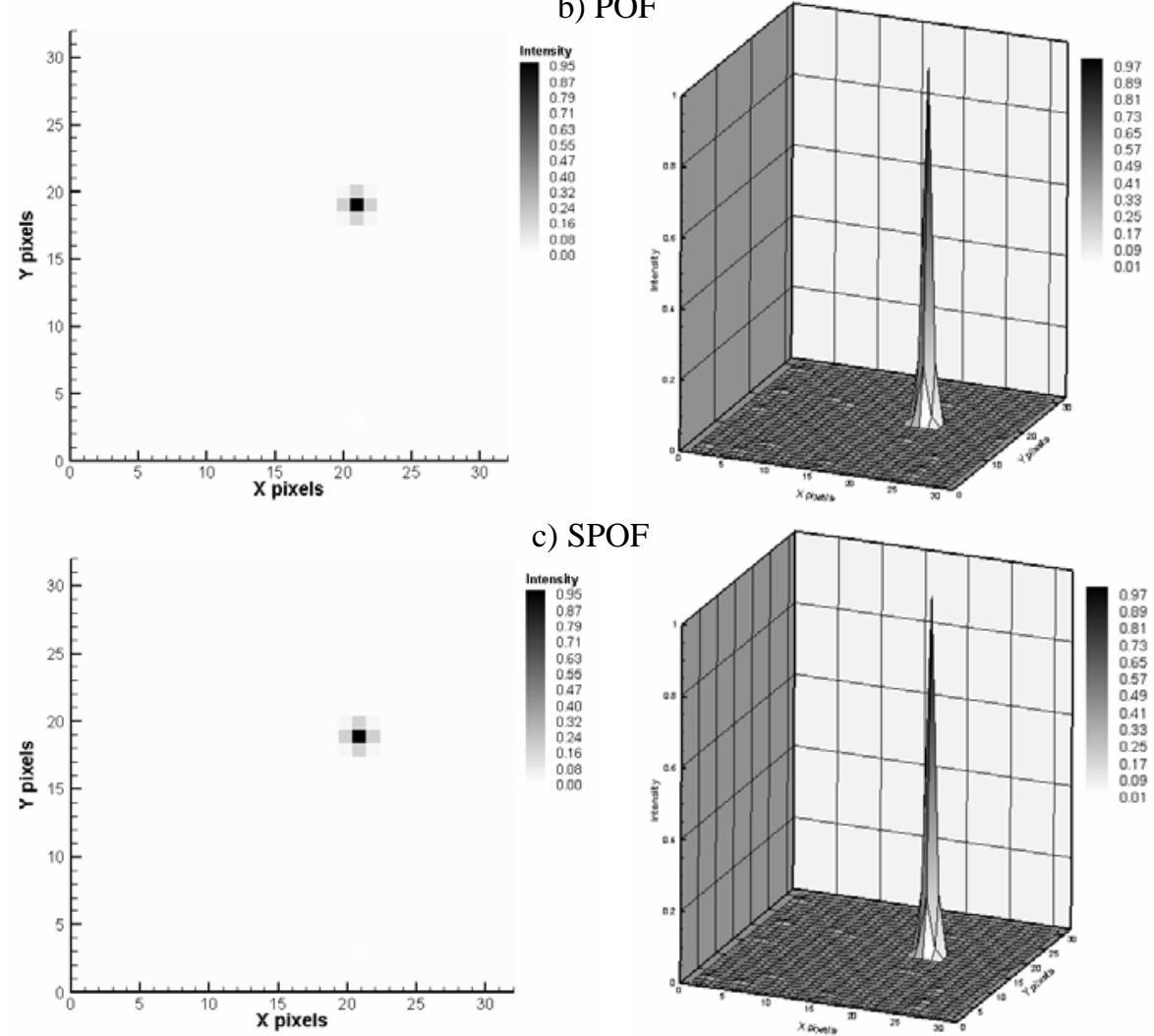

c) SPOF

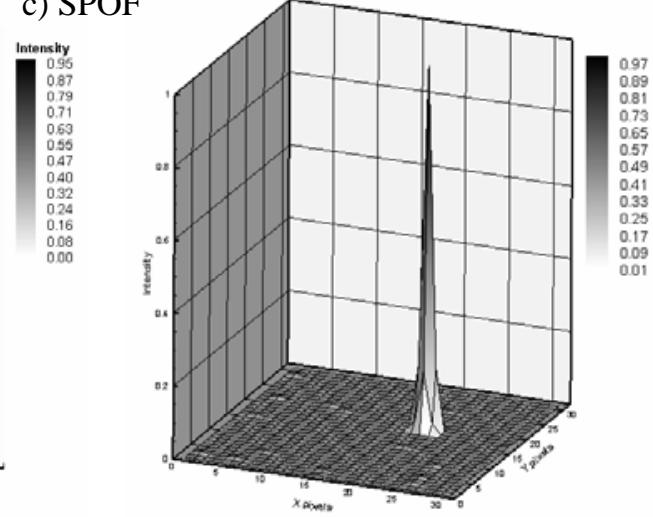

Figure 5: Case 1: Simulated PIV image data at top left. The output planes from the three filter functions are shown: a) MSF, b) POF, c) SPOF. The correlation peak in the MSF case is wider than the peak in the phase only filter cases. The sidelobes observed in the MSF case are significantly attenuated in the phase only filtering cases. 

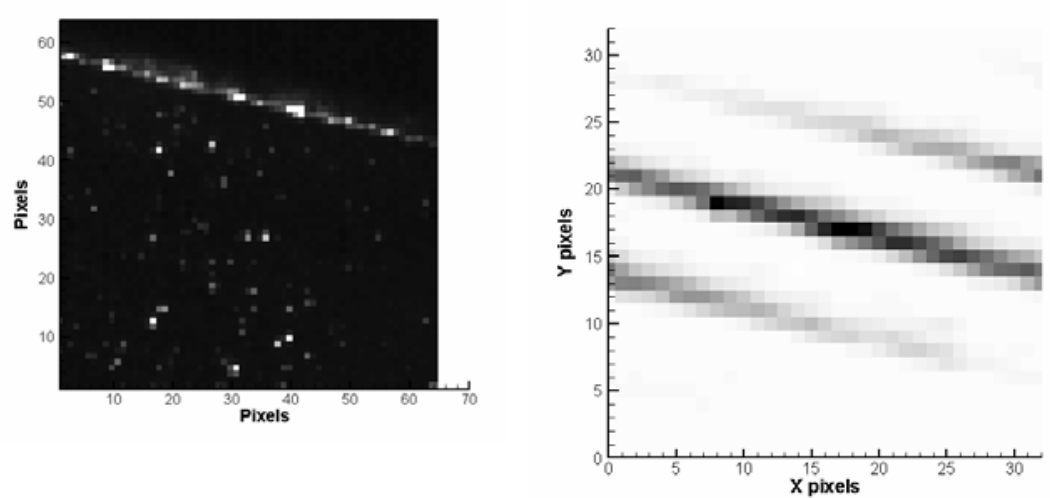

a) MSF
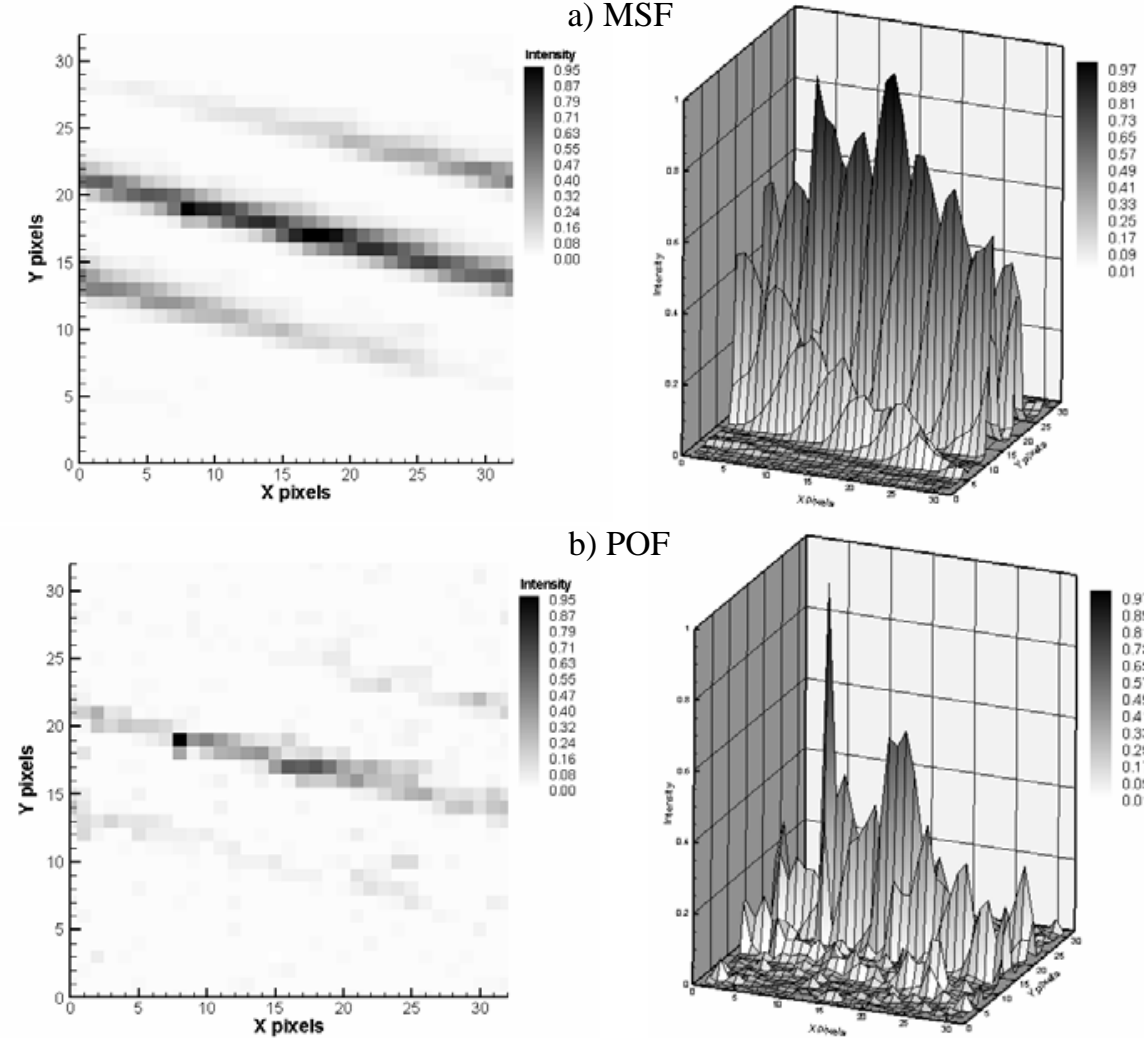

b) $\mathrm{POF}$
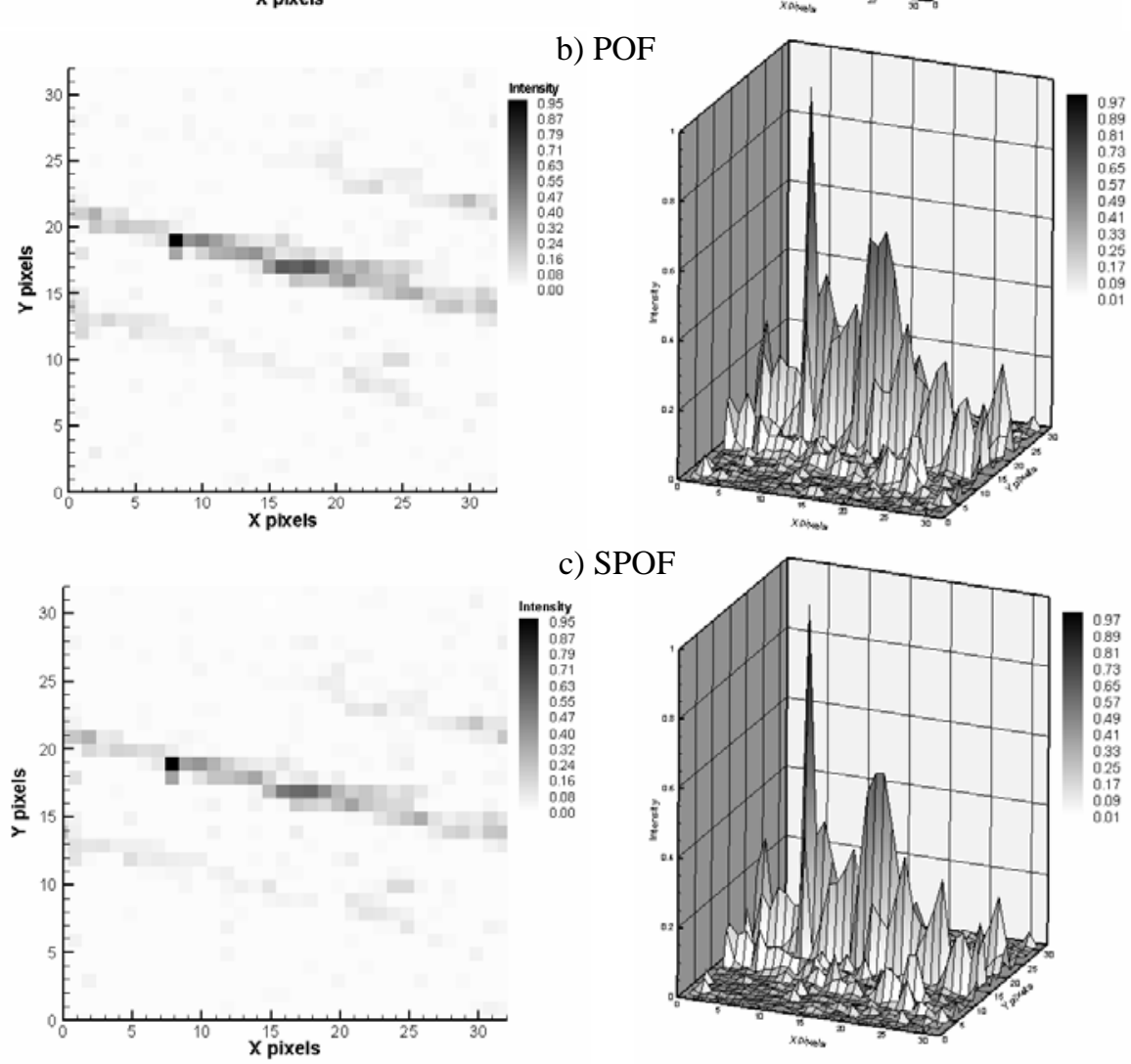

c) $\mathrm{SPOF}$

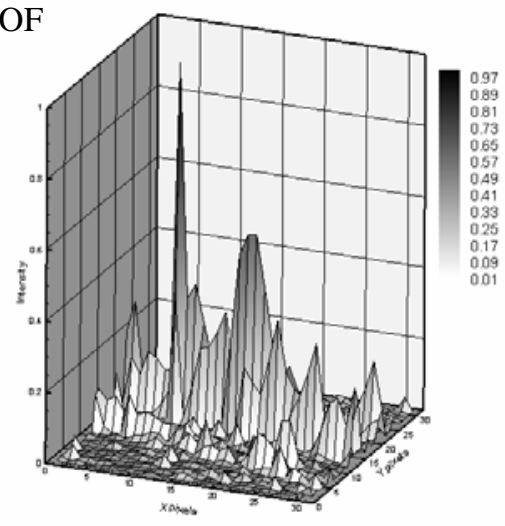

Figure 6: Case 2: Experimental PIV image data with a surface flare. The output planes from the three filter functions are shown: a) MSF, b) POF, c) SPOF. The particle displacement peak is not discernible in the MSF result. For the two phase only filtering techniques, the correlation peak is clearly visible above the noise generated from the surface flare light. 

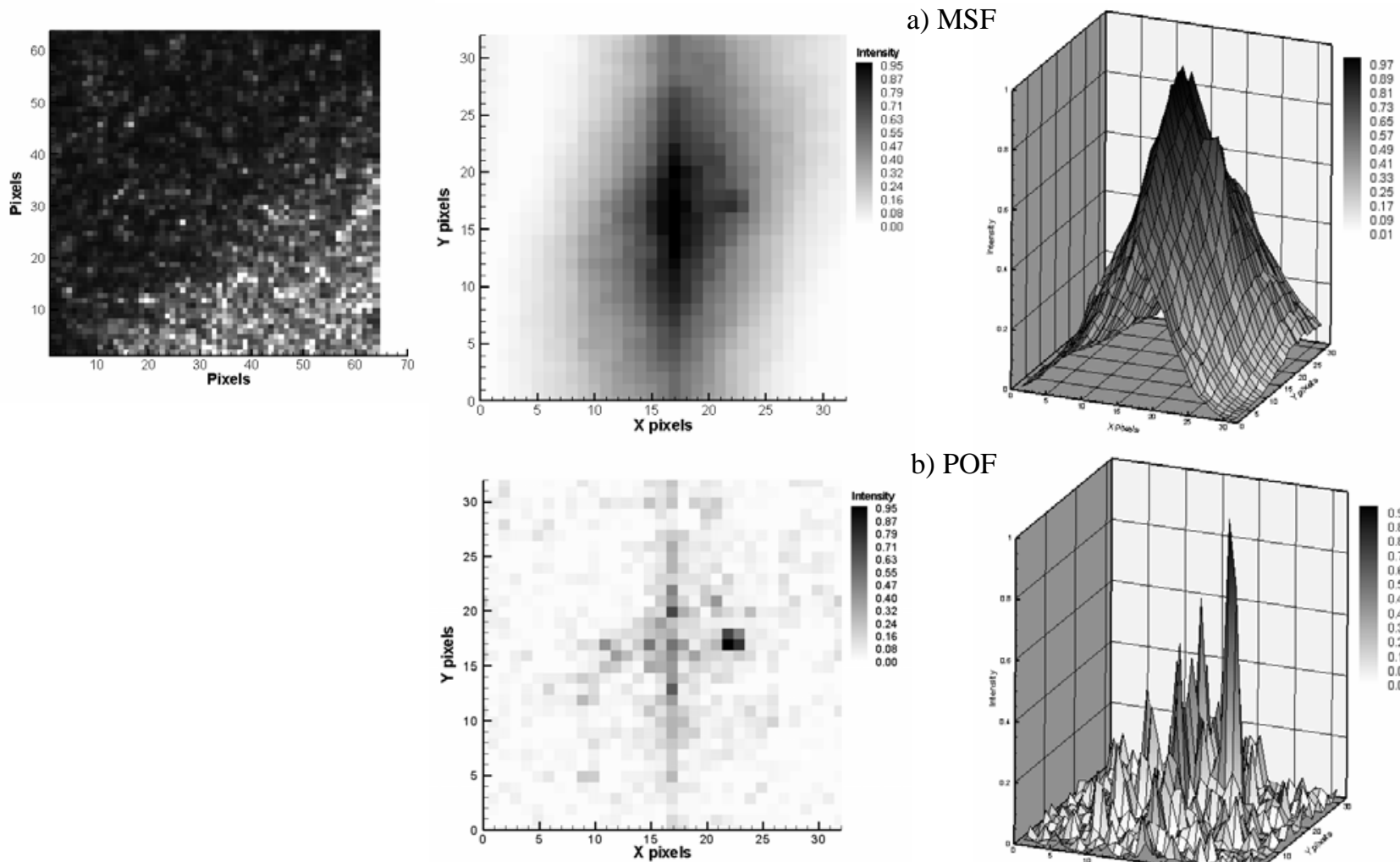

b) POF

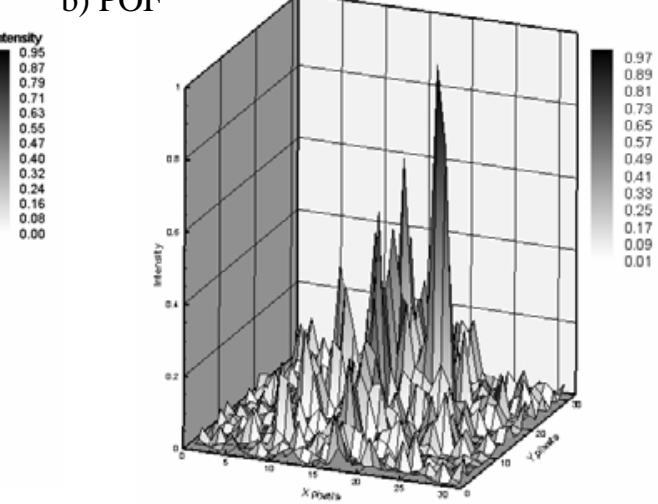

c) SPOF
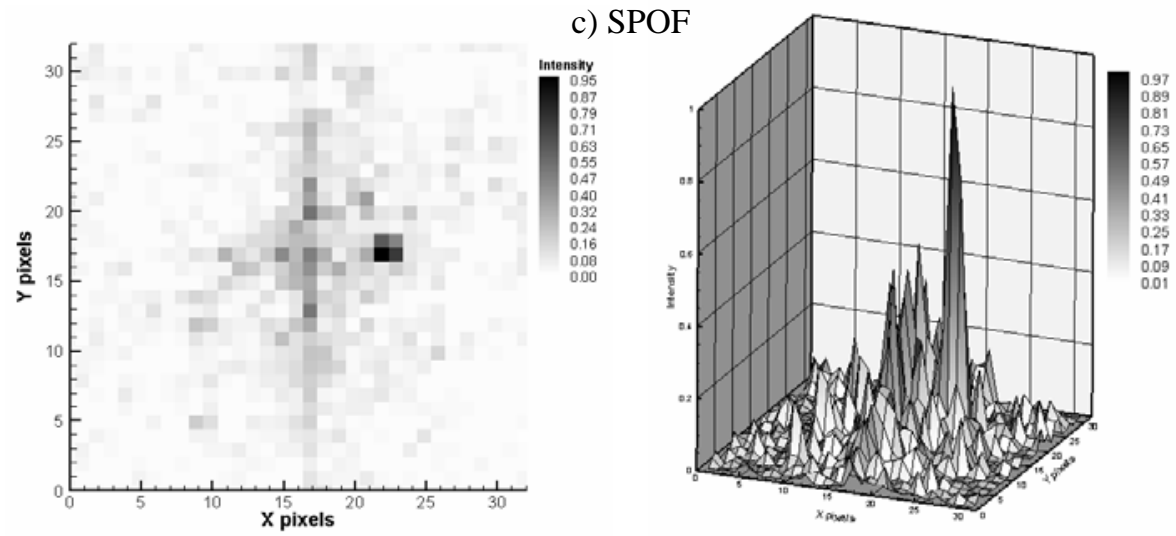

Figure 7: Case 3: Experimental PIV image with a flame front seeding interface shown at top left. The output planes from the three filter functions are shown: a) MSF, b) POF, c) SPOF. In the MSF result, the particle displacement peak is barely visible next to the large dc peak generated by the seeding interface in the subregion. The two phase only filtering techniques produce sharp correlation peaks readily identifiable above the background noise. 

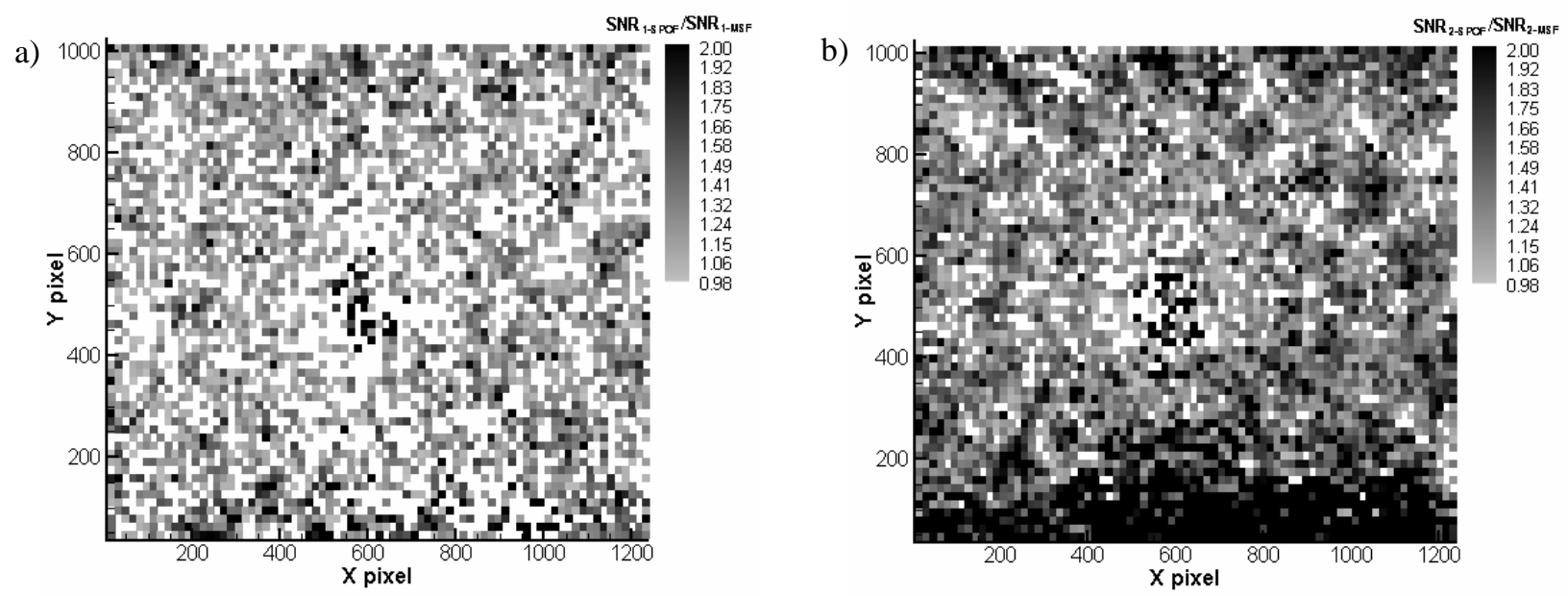

Figure 8: Ratio of SNR values for the DPIVChallenge vortex image for a) Ratio of $\mathrm{SNR}_{1}$ values for SPOF/MSF and b) Ratio of $\mathrm{SNR}_{2}$ values for SPOF/MSF case. The pixilated gray scale maps have all values below 0.98 set to pure white and all values above or equal to 2 set to pure black. Each "pixel" in the plots corresponds to a correlation subregion. The majority of the time, the SPOF technique produces higher SNR values than the MSF technique (fewer white pixels).

a)

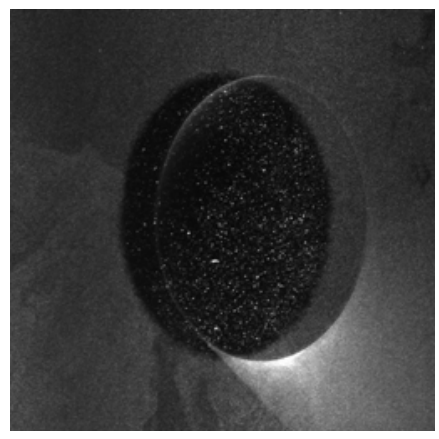

b)

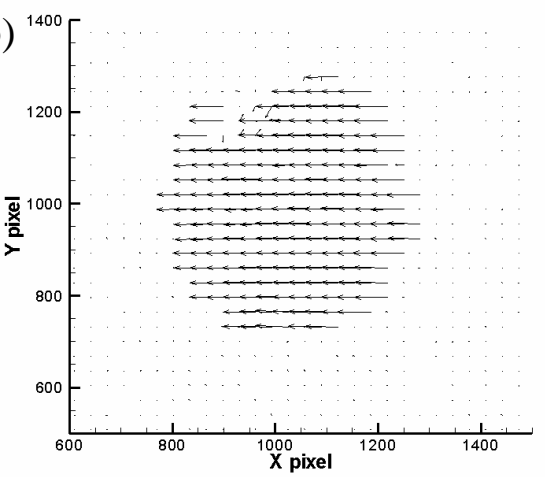

c)

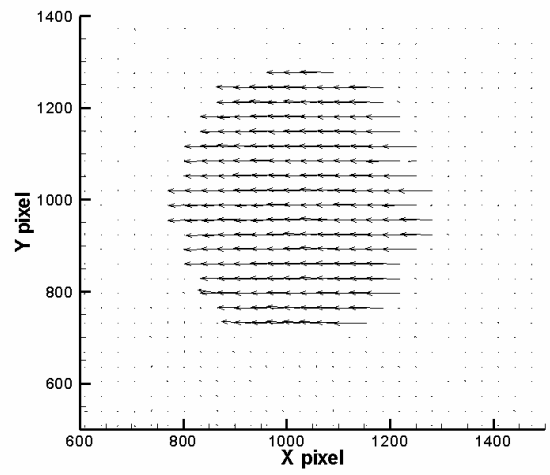

Figure 9: a) An image from the left camera in a stereo DPIV setup, where the light sheet is oriented perpendicular to the nozzle flow. Flare light from the nozzle contaminates the PIV processing. b) Standard cross-correlation processing of the PIV image data yields spurious vectors at the top of the nozzle. c) SPOF processing of the PIV image data yields the true velocity vector field, the effect of the flare light is removed.
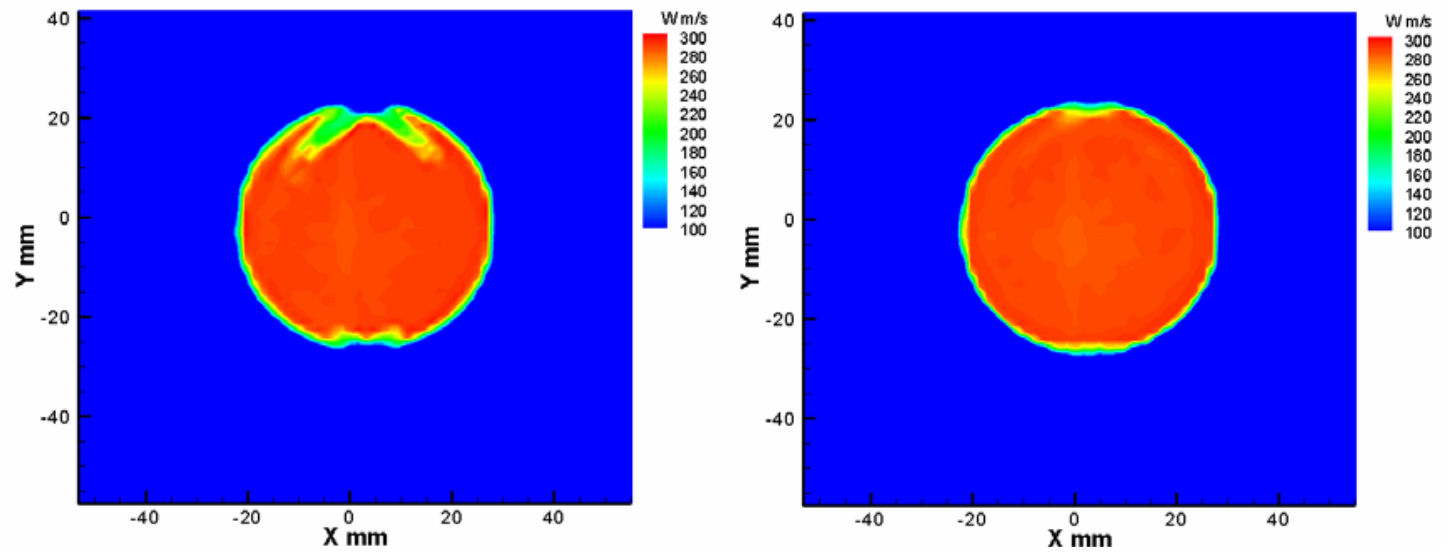

Figure 10: Ensemble averaged, 3-D velocity field where only the w-component of velocity is plotted here as a color contour plot. The model flare light contaminated velocity vector maps from the left and right camera views are combined in the computed 3-component velocity field resulting in the spurious velocities at the top and bottom of the nozzle flow field. These are manifested as the green arcs at the nozzle flow top and bottom centerline. Using the SPOF processing of the same image data yields the results on the right, where no evidence of the model flare light contamination is observed in the final result. 


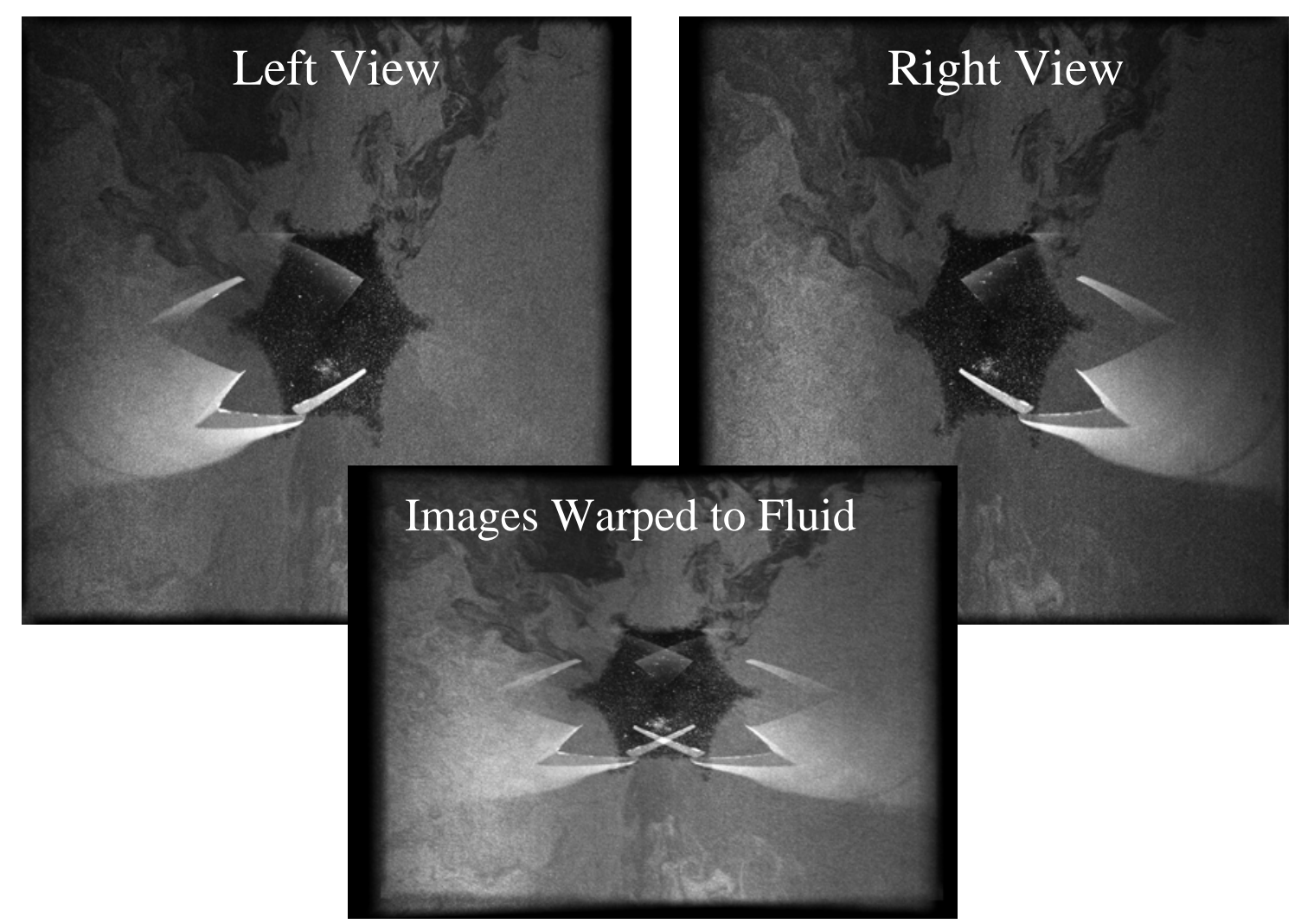

Figure 11: A third example of the benefits of the phase only filtering techniques. This data is from a chevron nozzle flow experiment where the stereo PIV system is again configured for cross-stream measurements. The left and right camera views are shown along with the overlapped images warped back to the fluid space.

\section{SPOF Not Used}

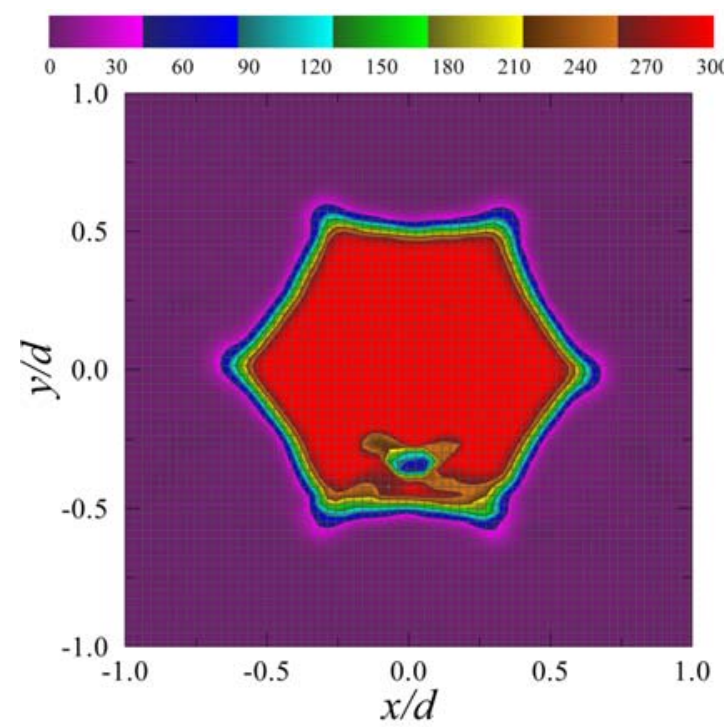

\section{SPOF Activated}

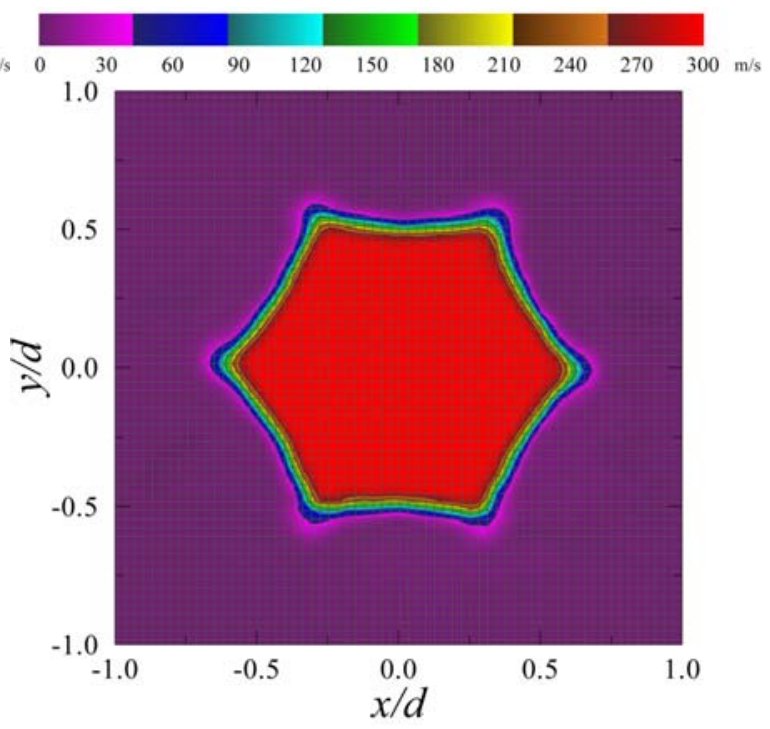

Figure 12: Ensemble averaged, 3-D velocity field where only the w-component of velocity is plotted here as a color contour plot. The model flare light contaminated velocity vector maps from the left and right camera views are combined in the computed 3-component velocity field resulting in the spurious velocities at the bottom of the nozzle flow field. Using the SPOF processing of the same image data yields the results on the right, where no evidence of the model flare light contamination is observed in the final result. 

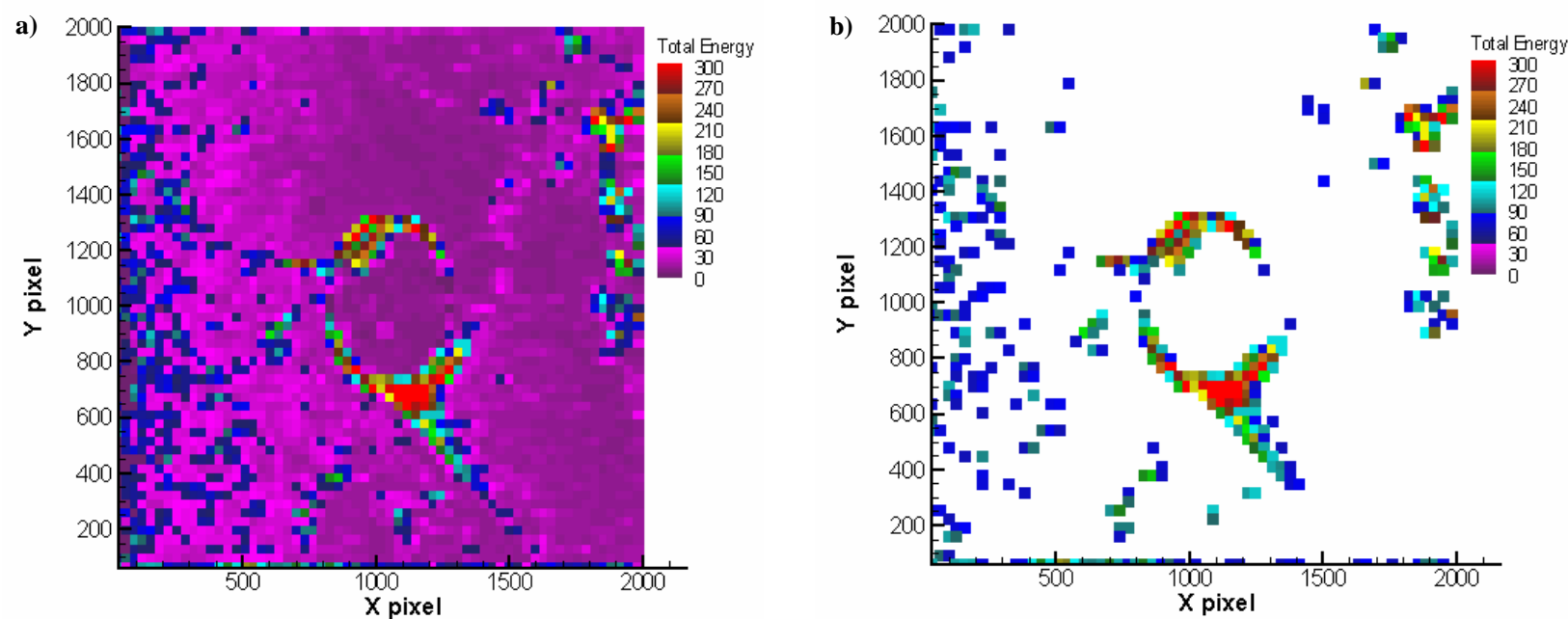

Figure 13: Plots of the total energy on the correlation plane for the axi-symmetric nozzle image set. a) Contour map of total energy, where red colored regions indicate areas where the model flare light is high; b) Same plot as in a), except a threshold level of 64 has been applied. Only high energy regions, where the SPOF should be applied, are now depicted.

a)

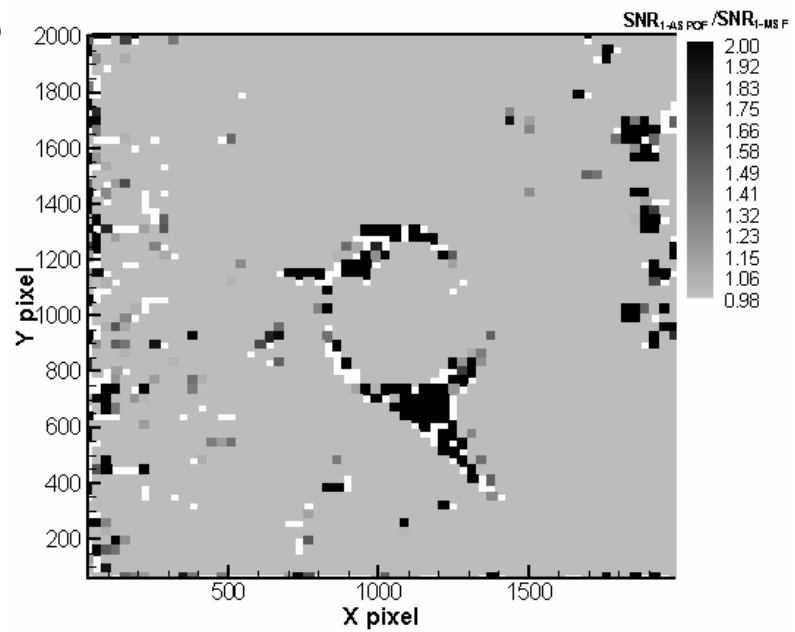

b)

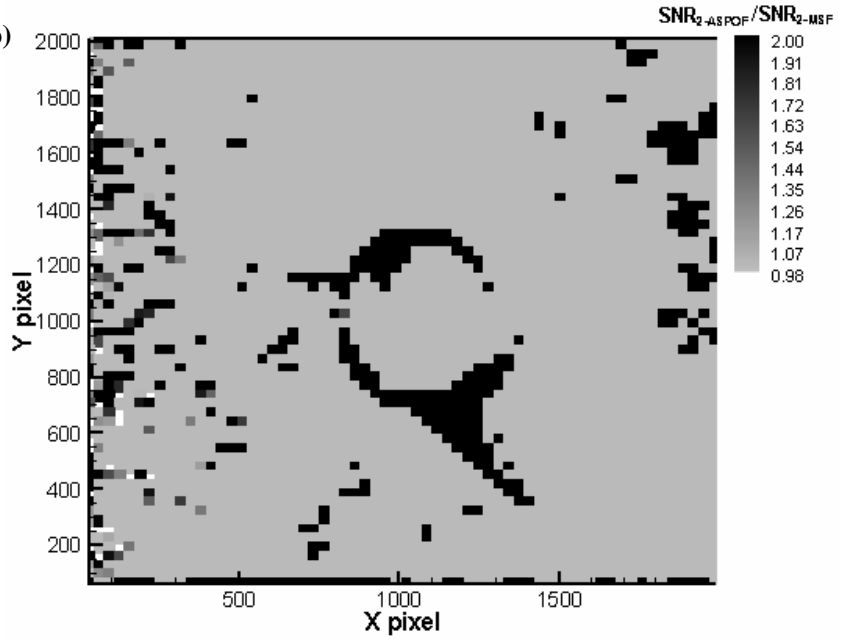

Figure 14: Ratio of the SNR values for the axi-symmetric nozzle flow case for a) Ratio of $\mathrm{SNR}_{1}$ values for ASPOF/MSF, b) Ratio of $\mathrm{SNR}_{2}$ values for ASPOF/MSF. In these pixilated contour plots, values below 0.98 are clipped (set to white). In the Automatic SPOF technique, the MSF is used to process the entire image, yielding unity ratio in the SNR values nearly every where (light gray areas). The SPOF is only applied when the total energy exceeds the threshold level of 16, as shown in figure $13 \mathrm{~b}$. The $\mathrm{SNR}_{1}$ values are nearly always larger for the ASPOF. Similarly, the $\mathrm{SNR}_{2}$ values generally exceed those obtained from the MSF.
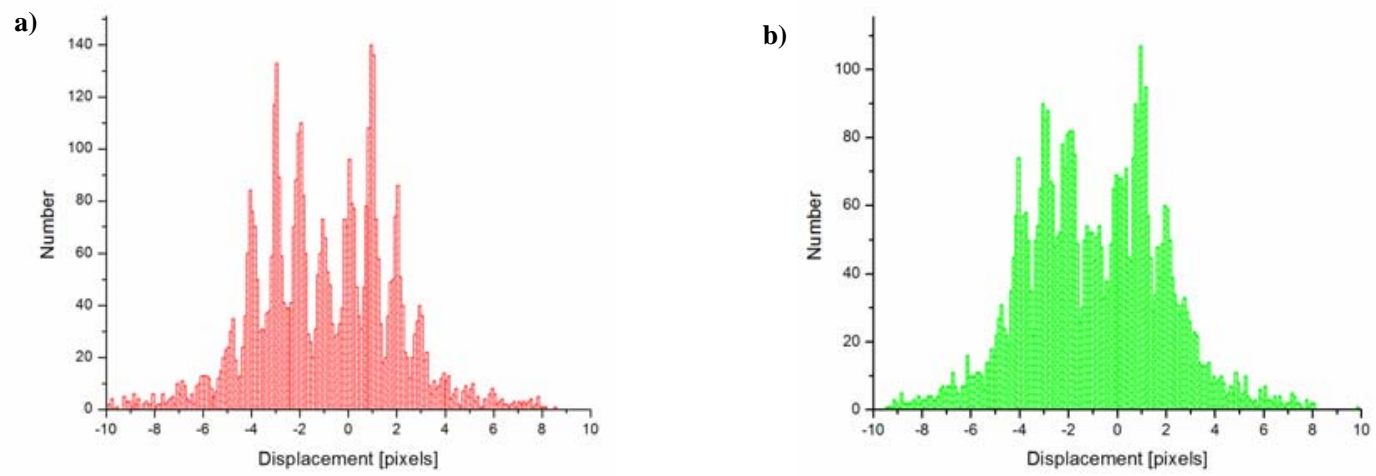

Figure 15: Comparison of peak locking between the a) MSF and b) SPOF. The MSF case exhibits more peak locking than the symmetric phase only filtering technique. 